\title{
Design and Implementation Guidelines for a Modular Spectral-Domain Optical Coherence Tomography Scanner
}

\author{
Farid Atry $\mathbb{D}^{1},{ }^{1}$ Israel Jacob De La Rosa $\mathbb{D},{ }^{2}$ Kevin R. Rarick $\mathbb{D}^{,},{ }^{3}$ and Ramin Pashaie $\mathbb{D}^{2}$ \\ ${ }^{1}$ Department of Biomedical Engineering, University of Wisconsin-Madison, Madison, WI, USA \\ ${ }^{2}$ Electrical Engineering Department, University of Wisconsin-Milwaukee, Milwaukee, WI, USA \\ ${ }^{3}$ Department of Pediatrics, Medical College of Wisconsin, Milwaukee, WI, USA \\ Correspondence should be addressed to Ramin Pashaie; pashaie@uwm.edu
}

Received 30 November 2017; Accepted 4 January 2018; Published 12 February 2018

Academic Editor: Chenggen Quan

Copyright ( 2018 Farid Atry et al. This is an open access article distributed under the Creative Commons Attribution License, which permits unrestricted use, distribution, and reproduction in any medium, provided the original work is properly cited.

\begin{abstract}
In the past decades, spectral-domain optical coherence tomography (SD-OCT) has transformed into a widely popular imaging technology which is used in many research and clinical applications. Despite such fast growth in the field, the technology has not been readily accessible to many research laboratories either due to the cost or inflexibility of the commercially available systems or due to the lack of essential knowledge in the field of optics to develop custom-made scanners that suit specific applications. This paper aims to provide a detailed discussion on the design and development process of a typical SD-OCT scanner. The effects of multiple design parameters, for the main optical and optomechanical components, on the overall performance of the imaging system are analyzed and discussions are provided to serve as a guideline for the development of a custom SD-OCT system. While this article can be generalized for different applications, we will demonstrate the design of a SD-OCT system and representative results for in vivo brain imaging. We explain procedures to measure the axial and transversal resolutions and field of view of the system and to understand the discrepancies between the experimental and theoretical values. The specific aim of this piece is to facilitate the process of constructing custom-made SD-OCT scanners for research groups with minimum understanding of concepts in optical design and medical imaging.
\end{abstract}

\section{Introduction}

Spectral-domain optical coherence tomography (SD-OCT) [1] is a versatile imaging technique with the ability to acquire 3D images at a resolution as low as $2 \mu \mathrm{m}$ up to several hundred micrometers deep in a scattering tissue. Optical coherence tomography can visualize the detailed structure of different biological tissue samples [2] such as brain cytoarchitecture [3], large cellular structures (e.g., $[4,5])$, or retinal layers in the eye $([6-8]$ to name a few) or functional retinal images (e.g., $[9,10])$. In addition, it can provide functional information by capturing the vascular morphology through optical coherence angiography (e.g., [11-14]) or by recording vascular blood velocity (e.g., [15-18]). Spectroscopic OCT [19] can provide additional functional information by analysis of light absorption within the bandwidth of the light source $[20,21]$. Polarization-sensitive OCT (PS-OCT) [2224] analyzes the effect of tissue on the light polarization to provide a contrast mechanism other than light intensity. Different implementation of PS-OCT has been proposed for SD-OCT structure in the past years [25-28]. Optical coherence elastography (OCE) is another application of OCT imaging, which aims to study the mechanical properties of the tissue. OCE, which was originally based on ultrasonic elastography, uses optical coherence imaging to analyze tissue displacement when it is vibrated, stretched, or compressed $[29,30]$. This concept was also adapted and used for SDOCT imaging (e.g., [31, 32]). For most OCT applications, the imaging tasks can be performed without the use of exogenous contrast agents, and in many cases it can be considered as a noninvasive imaging technique. However, OCE requires some external source of force to create vibration 
or mechanical stress in the tissue. In some applications, spectroscopic OCT is performed when external agents such as gold nanoparticles are introduced to the tissue to improve imaging signal-to-noise ratio (SNR). Except for polarizationsensitive OCT, the other abovementioned OCT applications are possible with a typical SD-OCT platform similar to what is discussed in this paper. For PS-OCT, some modifications to the system are necessary to precisely control the polarization of incident light and to detect the polarization state of the backscattered light. However, the authors only used the suggested design for Doppler OCT and optical coherence angiography imaging. We refer interested readers to $[33,34]$ for a more detailed discussion on each of these techniques. Nonetheless, a broader range of research questions can be addressed by combining SD-OCT imaging techniques with other modalities such as fluorescence microscopy or electrophysiology. These beneficial features have promoted SD-OCT applications and as a result an increasing number of commercial SD-OCT scanners have become available for basic science and clinical research applications. It is important to note other OCT configurations (swept-source OCT and time-domain OCT) that are also widely used and are capable of performing these tasks. However, in this article, the focus is on developing a guideline for a SD-OCT device.

Despite commercial developments of OCT scanners and the wide range of applications, this technology is not readily accessible to many research groups either due to the cost considerations or due to the difficulty of modifying commercial systems to meet evolving research goals. The necessary modifications are sometimes impossible to achieve or require complex optical designs or custom-machined parts which make the implementation process slow and increase the ultimate cost of the SD-OCT setup. Design and development of a custom-made OCT scanner is potentially a viable solution in such cases; however, it can also be a time-consuming process when users lack experience or knowledge in the field of optical engineering. A discussion on the optical design and practical implementation of a custom SD-OCT system can be beneficial to research groups with different backgrounds and with no or minimal knowledge of the science of optics to allow them to construct their in-house OCT system that can be tailored to their specific research needs. This can greatly accelerate research throughput of many laboratories and is the specific aim of the current manuscript.

While the theory and applications of SD-OCT are vastly presented in many great literatures (e.g., [2, 35-44]), the lack of a detailed discussion on optical design and practical implementation guidelines of this device precludes the utilization of SD-OCT scanners by many researchers. In this paper, we review the fundamental concepts of SD-OCT image formation and propound practical considerations for the design and implementation of the device, along with some guidelines for choosing proper components to obtain the desired image quality. We offer a procedure to build a cost-effective SD-OCT imaging system versatile enough to be integrated with other optical technologies. The proposed system is portable and easy to disassemble/reassemble, which facilitates its transportation. Except for a few simple custommade optical mounts, it mainly utilizes off-the-shelf optical components, which makes the implementation more cost and time efficient.

\section{Materials and Methods: Theory}

OCT was proposed as an extension of the low-coherence reflectometry in 1991 by Huang et al. [47]. In their system, they coupled a low-coherence light source to a fiber-based Michelson interferometer. The Michelson interferometer splits the beam into two different paths: a reference and a sample path. The sample path illuminates a sample and collects the backscattered light. The reference path guides the light toward a reference mirror that moves back and forth to continuously modulate the optical length of the reference path. Light that is received back from the sample and the reference mirror is combined and guided toward a photodetector. When the optical lengths of the sample and reference path are almost equal (i.e., the difference is no more than the source coherence length), the interference pattern becomes visible. The amplitude of the interference is maximum when the lengths of the two paths are exactly equal and drops as we move the reference mirror out of the coherence length. It is theoretically and experimentally proven that the axial (along the $z$ direction) resolution of such an OCT scanner is the same as the coherence length of the source $\left(l_{c}\right)$ [48]. For a Gaussian light source which produces the central wavelength of $\lambda_{0}$ and the bandwidth of $\Delta \lambda$, the coherence length is $l_{c}=\ln (2)(2 / \pi)\left(\lambda_{0}^{2} / \Delta \lambda\right)$. This assembly was the first generation of an OCT imager, which is usually called a time-domain OCT (TD-OCT). The main drawback of this scanning mechanism is the need to physically move the reference mirror, which limits the speed and performance of the scanner. Development of the frequency-domain OCT technology was basically the attempt to overcome this limitation. Frequency-domain OCT devices generate images by analyzing the spectral pattern of the collected interferograms generated by combining the reflected lights from the reference and the sample arms. In frequency-domain OCT scanners, the spectrum of the interference pattern is produced by either replacing the photodetector in the conventional TD-OCT by a spectrometer in spectral-domain OCT configurations or utilizing a light source that rapidly sweeps the illumination wavelengths over a desired bandwidth in swept-source OCT (SS-OCT) devices. TD-OCT is relatively of low cost; however, the requirement for scanning path length in the reference arm severely limits the maximum speed to be typically less than a few thousand A-scans per second. Some research groups have been able to achieve scan rates as fast as $20 \mathrm{~K}$ A-scans per second [49]. SD-OCT can achieve imaging speed that is almost an order of magnitude faster than TD-OCT. The system is more complex and requires the design and implementation of a fast spectrometer. Therefore, the complexity and cost of a SD-OCT scanner are higher than of a TD-OCT device. SS-OCT can achieve scan rates faster than SD-OCT. By the use of signal multiplexing or full-field recording, this method can increase the imaging speed even beyond this level. A-scan rates beyond $200 \mathrm{~K}$ lines per second are reported for SS-OCT. While SD-OCT 
is typically slower than SS-OCT, it offers superior phase stability and axial resolution, which is beneficial in Doppler flowmetry or capillary imaging that require high phase stability and fine axial resolution. The current SS-OCT technology provides fast imaging capability with large imaging depth.

Here, we discuss the theory as well as design and implementation guidelines for a typical fiber-based spectraldomain OCT. Some statements are also valid for other OCT configurations.

In 1995, Fercher et al. proposed a spectral interferometry technique, based on the Fourier diffraction theorem, to measure the optical length of objects along the light beam (the $z$-axis) [1]. For weakly scattering objects, where the first-order Born approximation is valid, they demonstrated that, under low-coherence illumination, the spectrum of the backscattered light is proportional to the Fourier transform of the scattering potential of the sample $(F(z))$. Their work can be considered as the foundation of Fourier domain OCT systems. For this discussion, we adapt their approach to estimate the backscattered electric field from a sample when it is exposed to a monochromatic Gaussian beam of light with power spectral density of $P_{S}(k)$, where $k=\left|\mathbf{k}_{i}\right|=\left|\mathbf{k}_{s}\right|=2 \pi / \lambda$ is the wavenumber and $\mathbf{k}_{i}$ and $\mathbf{k}_{s}$ are the wave vectors of the illuminating and backscattering waves, respectively. When assuming that the origin of the coordinate system is placed at the surface of a specimen of thickness $T$, the backscattered electrical field $\left(E_{\text {Sample }}^{D}\right)$ at a sufficiently large distance $D$ along the illumination axis is estimated by [1]

$$
\begin{aligned}
E_{\text {Sample }}^{D}(\mathbf{k}, t)= & \alpha \sqrt{P_{S}(k)} e^{(i k \cdot D-i \omega t)} \\
& \times \int_{0}^{T} F\left(z^{\prime}\right) e^{-i\left(\mathbf{k}_{s}-\mathbf{k}_{i}\right) z^{\prime}} d z^{\prime} \\
= & \alpha \sqrt{P_{S}(k)} e^{(i k \cdot D-i \omega t)} \times \operatorname{FT}\{F(z)\},
\end{aligned}
$$

where FT is the Fourier transform with respect to $z, \omega$ is the angular frequency of the monochromatic light, and $\alpha$ is a constant depending on the distances and configuration of the beam. When this beam interferes with the beam from the reference mirror which is placed at the location $0\left(E_{\mathrm{Ref}}^{D}\right)$, the combined electric field is

$$
\begin{aligned}
E_{\text {total }}^{D}(\mathbf{k}, t)= & E_{\text {Sample }}^{D}(\mathbf{k}, t)+E_{\text {Ref }}^{D}(\mathbf{k}, t) \\
= & \alpha \sqrt{P_{S}(k)} e^{(i k \cdot D-i \omega t)} \times \operatorname{FT~}\{F(z)\} \\
& +\beta \sqrt{P_{S}(k)} e^{(i k \cdot D-i \omega t)} \times \operatorname{FT}\{R \delta(z)\} .
\end{aligned}
$$

The parameter $R$ is the reflectivity of the mirror and $\beta$ is the portion of the back-reflection light that is used to produce interference. The mirror is assumed to be infinitely thin so its reflection can be modeled as a Dirac delta function at $z=0$ $(\delta(z))$. The intensity of the interfering signal is the square of the absolute value of this electric field:

$$
\begin{aligned}
I(k)= & E_{\text {total }}^{D}(\mathbf{k}, t) E_{\text {total }}^{D}(\mathbf{k}, t)^{*} \\
= & P_{S}(k) \alpha^{2}|\mathrm{FT}\{F(z)\}|^{2}+P_{S}(k) \beta^{2}|\mathrm{FT}\{R \delta(z)\}|^{2} \\
& +P_{S}(k) \alpha \beta^{*} \mathrm{FT}\{F(z)\} \operatorname{FT}\{R \delta(z)\}^{*} \\
& +P_{S}(k) \alpha^{*} \beta \mathrm{FT}\{F(z)\}^{*} \operatorname{FT}\{R \delta(z)\},
\end{aligned}
$$

where the superscript $*$ represents the complex conjugate. In general, if the reflection from the mirror is significantly stronger than the backscattering from the sample, then the first term $\left(S(k) \alpha^{2}|\mathrm{FT}\{F(z)\}|^{2}\right)$ has a negligible effect and can be ignored. Therefore, the intensity of interfering light at wavenumber $k$ can be approximated by

$$
\begin{aligned}
I(k) \approx & P_{S}(k) \beta^{2}|\mathrm{FT}\{R \delta(z)\}|^{2} \\
& +P_{S}(k) \alpha \beta^{*} \operatorname{FT}\{F(z)\} \operatorname{FT}\{R \delta(z)\}^{*} \\
& +P_{S}(k) \alpha^{*} \beta \operatorname{FT}\{F(z)\}^{*} \operatorname{FT}\{R \delta(z)\} .
\end{aligned}
$$

By measuring the interference intensity at multiple wavenumbers and applying an inverse FT to $I(k)$, we obtain the scattering potential of the tissue along the $z$-axis $(F(z))$ :

$$
\begin{aligned}
\operatorname{IFT}\{I(k)\} \approx & \operatorname{IFT}\left\{\beta^{2} P_{S}(k)\right\} \star R^{2} \delta(z) \\
& +\operatorname{IFT}\left\{\alpha \beta P_{S}(k)\right\} \star F(z) \star R \delta(-z) \\
& +\operatorname{IFT}\left\{\alpha \beta P_{S}(k)\right\} \star F(-z)^{*} \star R \delta(z) .
\end{aligned}
$$

The operator $\star$ is a convolution operator. Here, we assumed that $\alpha, \beta$, and $R$ are real numbers independent of the wavenumber. If $P_{S}(k)$ is a smooth function of the wavenumber, then its IFT is mostly centered at the origin and can be approximated by a Dirac delta function $\delta(z)$ :

$$
\begin{aligned}
\operatorname{IFT}\{I(k)\} \approx & \beta^{2} R^{2} \delta(z)+\alpha \beta R \times F(z)+\alpha \beta R \\
& \times F(-z)^{*} .
\end{aligned}
$$

By placing the origin of the coordinate system on the tissue surface, we know the sample is confined to positive $z$ values. Hence, $A(z)=\operatorname{IFT}\{I(r, k)\}$ on the positive $z$-axis represents the scattering potential of the object which is called the depth profile. $A(z)$ is a Hermitian function of $z$, and on the negative $z$-axis, $A(z)$ is the complex conjugate of its values on the positive axis.

In practice, the light source has a limited bandwidth which causes $\operatorname{IFT}\left\{P_{S}(k)\right\}$ to have a finite width. This width is the axial resolution of the spectral-domain OCT imaging. For a given power spectral density of $P_{S}(k)$, it is possible to use the Fourier theorem to calculate the axial resolution of the OCT imaging. For a light source with a Gaussian spectrum of bandwidth $\Delta \lambda$ at the central wavelength of $\lambda_{0}$, the axial resolution is

$$
\delta z=\frac{2 \ln (2)}{\pi} \frac{\lambda_{0}^{2}}{\Delta \lambda}=l_{c} .
$$


The theoretical resolution in spectral-domain OCT imaging is the same as the coherence length of the light source $\left(l_{c}\right)$. If $\alpha$ and $\beta$ are not uniform across the light spectrum, the axial resolution can be larger than (7). The lateral resolution in an OCT imaging system is a function of the central wavelength and the numerical aperture of the imaging system and is decoupled from its axial resolution. For more in-depth discussions and analysis of OCT image formation, we refer the readers to many articles and books including but not limited to [33, 35-43, 50-53].

In spectral-domain OCT imaging, the depth of the scatterer and the interference pattern are related via Fourier transform (see (6)). Therefore, when the depth of a scatterer $(z)$ increases, its corresponding frequency in the interference pattern $(I(k))$ also increases. The spectrometer, however, acquires a finite number of samples from the interference pattern and, in practice, a discrete Fourier transform (DFT) is used to convert the interference pattern to a depth profile. According to the Nyquist sampling theorem, the spectral resolution of the spectrometer determines the maximum detectable frequency in the interference pattern $(I(k))$ and, hence, the imaging depth. When a scatterer falls outside this imaging range, it produces an interference pattern with a frequency larger than the Nyquist limit of the sampling rate. Due to aliasing, the depth of such a sample is misrepresented in OCT images. If the number of pixels on the camera is $N$ and the measured spectral bandwidth (in wavenumber) is $\Delta \kappa$, the spectrometer's sampling interval is estimated by $|\delta \kappa|=|\Delta \kappa / N| \approx\left(2 \pi / \lambda_{0}^{2}\right)(\Delta \lambda / N)$. Therefore, according to (6), the imaging range of the SD-OCT device is $[36,50,52]$

$$
\begin{aligned}
2 \times z_{\max } & =\frac{1}{2} \frac{1}{\left(1 / \lambda_{0}^{2}\right)(\Delta \lambda / N)}=\frac{\lambda_{0}^{2}}{2(\Delta \lambda / N)} \\
\text { or } z_{\max } & =\frac{N \lambda_{0}^{2}}{4 \Delta \lambda},
\end{aligned}
$$

where $z_{\max }$ is the maximum imaging depth without any aliasing effect. $z_{\max }$ is directly proportional to the number of pixels in the spectrometer. For a large imaging range, a detector with a sufficiently high number of pixels should be selected. According to (8), the imaging range can also be furthered by increasing the central wavelength or decreasing the bandwidth of the light source. However, either of these changes adversely affects the imaging resolution.

\section{Materials and Methods: Design Considerations}

3.1. Light Source. According to (7), a low-coherence light source is essential to obtain a fine axial resolution. Since the axial resolution of an OCT image is directly proportional to the square of the central wavelength of the light source and inversely proportional to its bandwidth, similar axial resolutions could be achieved in different regions of the spectrum. When using longer wavelengths, an increasingly wider light spectrum $(\Delta \lambda)$ is required to maintain the axial resolution. The main drawback of using longer wavelengths is the effect on the lateral resolution which is discussed later in this section. On the other hand, while shorter wavelengths can provide better lateral resolution, in majority of biological samples, the scattering effect increases at those wavelengths, which limits the effective imaging depth of an OCT. Therefore, the central wavelength of the light source should be chosen based on the application and the tissue of interest. For biological samples, both visible (e.g., [21, 54-57]) and near-infrared (e.g., [58-60]) wavelengths have been used. The high absorption coefficient of water in wavelength longer than $1 \mu \mathrm{m}$ justifies using short wavelength or visible light sources for transparent samples such as the eye. However, for typical brain imaging within the superficial $500 \mu \mathrm{m}$, a wavelength above $1000 \mathrm{~nm}$ is more popular.

The light source should be stable and should provide a low-noise output to obtain high quality images. Moreover, the emitted light should be spatially coherent. Superluminescent light emitting diodes (SLDs or SLEDs) are popular for spectral-domain OCT applications since they can provide low-noise and stable low-coherence light while they demand less maintenance in contrast to pumped lasers. With the recent advances in supercontinuum laser technology, the stability of this type of light sources has improved and their noise level has reduced. Supercontinuum laser sources provide light with an ultrawide spectral bandwidth and are used to obtain a fine axial imaging resolution. They provide more optical power in comparison with SLDs. For applications where high power and large light spectrum are needed, such light sources are a proper choice (e.g., [61, 62]). However, for applications that require high phase stability and low noise levels, such as Doppler velocimetry and OCT angiography, SLDs remain a superior choice compared to supercontinuum lasers. The spectral power distribution of the emitted light is also of great importance. A Gaussian-like power spectrum is ideal and provides an axial resolution close to the theoretical value. Deviation of the power spectrum from the Gaussian shape or sharp changes in the power spectrum can result in degradation of resolution or appearance of some vertical side lobes in OCT images. During postprocessing, some of these effects can be attenuated. Nonetheless, a Gaussian-like power spectrum is beneficial to achieve high quality OCT images.

3.2. Sample Arm. The design of a sample arm in an OCT system depends on the desired lateral resolution, maximum field of view, and the spectral bandwidth of the light source. Therefore, proper design and implementation of the sample arm are essential to achieve the desired specifications. For example, the axial resolution theoretically depends on the bandwidth of the light source; however, it still can be negatively influenced by a poor design of the sample arm if the chromatic aberrations cause significant spectral filtering in the sample arm [63]. A sample arm basically consists of four major components: a collimator, two galvanometric mirrors, intermediary optics, and an objective lens. Each component is influential in the specifications of the system.

3.2.1. Collimator. A collimator is a lens or lens mechanism that is placed in front of an optical fiber to produce a collimated beam. In combination with the rest of the arm, it forms an imaging system that images the fiber tip on 
the sample and, conversely, it images the focal plane of the objective on the tip of the optical fiber. In the forward path, when imaging the fiber on the sample, the collimated beam waist and the collimated beam quality have significant impact on the lateral resolution. The collimated beam radius depends on the numerical aperture of the optical fiber and also the effective focal length of the collimating lens system. When the fiber is placed at the focal plane of the collimator, the beam radius is approximated by

$$
w=f \cdot \theta_{\text {fiber }}=f \cdot \frac{\lambda}{\left(\pi w_{\text {fiber }}\right)},
$$

where $f$ is the effective focal length of the collimator and $w_{\text {fiber }}$ is the mode radius of the fiber at wavelength $\lambda$. The mode radius of a fiber is dependent on the wavelength and slightly changes across the spectrum of the wide-band source. In OCT applications, this dependency is neglected and the mode radius of the central wavelength is used in the calculation. By increasing the focal length of the collimator, it is possible to produce a larger collimated beam waist which leads to a smaller spot size on the sample (as discussed later in this article). When using a large focal length, the aperture of all intermediary optical components, including the galvanometric mirrors, needs to be selected accordingly to avoid beam truncation which causes signal loss or artifacts in the produced images.

In the return path, the collimator couples the photons, which are collected by the objective lens, to the single mode fiber. Optical aberrations or mismatch between the beam profile at the focal point of the collimator lens and the mode of the optical fiber causes loss in the signal and drop in image quality. The power loss is minimum when the returning beam waist matches the effective aperture of the collimator. Ideally, the collimator and the objective lens should be selected such that the loss due to mismatch between the collimator and objective lens aperture is minimum.

Optical aberrations in the collimating lens mechanism can degrade the quality of OCT images first by introducing imperfections to the collimated beam in the forward path, which can increase the sample spot size, and second by reducing the coupling efficiency to the fiber, which decreases the signal-to-noise ratio (SNR) in OCT images. Spherical and chromatic aberrations are two important imperfections that can be caused by the collimating lens system. A large spherical aberration reduces the lateral resolution of OCT images and the coupling efficiency of the backscattered photons. Longitudinal and lateral chromatic aberrations can cause the axial and lateral position of the focal spot to change as a function of wavelength. This affects the coupling efficiency across different wavelengths, which may degrade the axial resolution of the system [64] as well as its lateral resolution. Therefore, it is critical to select a proper collimator to achieve the desired imaging resolution and SNR.

A conventional biconvex or planoconvex lens may be able to produce the desired beam size; however, due to spherical and chromatic aberrations that are introduced by these types of lenses, the quality of the resulting beam is not satisfactory for high-resolution OCT applications. Typical achromatic doublets introduce slightly less chromatic and/or spherical aberrations; nonetheless, the remaining aberrations can still prevent achieving high lateral resolutions.

A better collimated beam quality can be achieved using an aspherical lens. Aspherical lenses are relatively more expensive yet usually designed to obtain short effective focal lengths. The quality of the Gaussian beam that is produced by this type of lenses is acceptable for most OCT applications. For extreme precisions, one can use a lens mechanism instead of a single lens; however, in most OCT applications, an aspherical lens can deliver the desired beam quality.

Besides the lens type, the placement of the fiber with respect to the lens can affect the quality of the beam. Small misalignments (tilt or misplacement) of the fiber and lens with respect to each other can also introduce aberrations to the beam and affect the OCT resolution. When using a lens with small aperture, such misalignments cause beam truncation, which results in loss of resolution and artifacts in the OCT images. Therefore, the fiber and collimating lens should be aligned very carefully. There are collimating packages that are offered by different vendors. These packages are designed in such a way that, for the designed wavelength and fiber radius, they can deliver a high quality beam without the need for fine alignment. Most collimating packages consist of a single aspheric lens. Some others, which are designed to produce high quality Gaussian beams ( $M$ factor close to 1 ), use a combination of multiple lenses to suppress aberrations. Typically, this type of collimator lenses can provide a better beam quality and higher coupling efficiency to the fiber, but they increase the cost of the system. When choosing a collimating package, it is important to select a package that is designed for angled physical contact (APC) to avoid artifacts in the OCT images due to back reflections at the fiber tip. This is discussed in more detail further in the paper.

Another important factor when choosing a collimator lens is its antireflection (AR) coating. Proper antireflection coating on the lenses in the sample arm, including the collimator, is important to acquire high quality OCT images as AR coating decreases the light reflection from the surface of the optical components and improves the image SNR. A good AR coating can reduce the reflection loss to as low as $0.2 \mathrm{~dB}$, while improper coating can result in a power loss up to $3 \mathrm{~dB}$ or higher. More importantly, the strong light reflection from surfaces which are not covered with proper AR coating introduces image artifacts as horizontal lines across the OCT images.

3.2.2. Galvanometric Mirrors. To obtain 2- or 3-dimensional images of a specimen, generally, the OCT beam raster-scans the tissue using a laser scanning mechanism (Figure 1). Two galvanometric mirrors rapidly scan the OCT beam spot on the sample along two orthogonal directions ( $x$ and $y$ ), while the depth profile at each $(x, y)$ location is obtained via low-coherence interferometry (see (6)). For fast and highresolution OCT imaging, it is necessary to use a galvanometric system which can achieve high scanning speeds with accurate control over the angle of the mirrors. Mechanical vibrations or timing jitter in the mirrors can affect the accuracy of OCT images or can produce artifacts in Doppler 


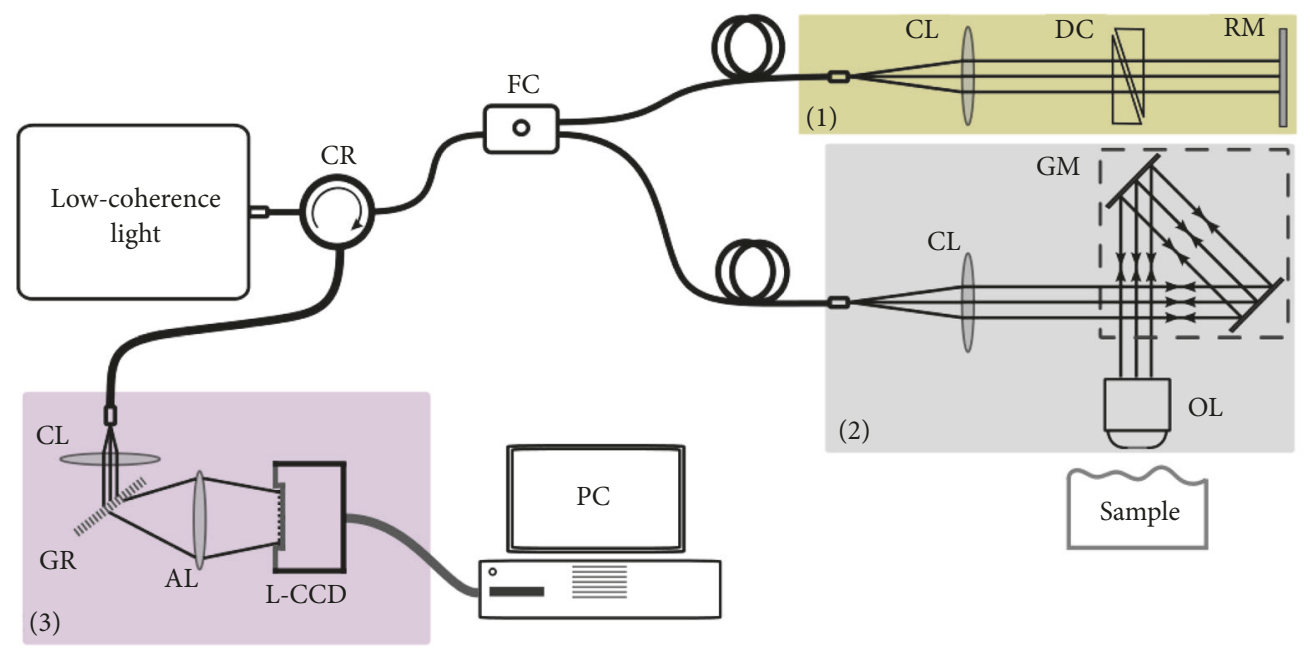

CL: collimating Lens
DC: dispersion compensator
RM: reference mirror
GM: galvanometer mirror
CR: fiber circulator
FC: fiber coupler

OL: objective lens AL: infrared achromatic lens L-CCD: line CCD camera GR: transmission grating PC: personal computer
(1) Reference arm

(2) Sample arm

(3) Spectrometer

FIGURE 1: An optical fiber-based spectral-domain OCT scanner. The system uses a low-coherence light source to launch light into both sample (Path 2) and reference (Path 1) arms. In the sample arm, a set of galvanometric mirrors raster-scan the sample under test. Light that is collected back from the reference and sample arms recombines to form interference patterns which are detected by the spectrometer (Path 3 ).

measurements and angiography images. Therefore, a servo circuitry which is equipped with a closed-loop controller is essential to achieve high stability and accuracy in positioning and scanning speed of the mirrors. The size of the mirrors affects the maximum speed that the galvanometric scanner can achieve. Small mirrors make it possible for the scanner to operate at a higher speed; however, the mirrors must be sufficiently large to avoid beam truncation. In contrast, since the OCT resolution depends on the collimated beam waist, the OCT beam cannot be too small. When a small beam and fine OCT lateral resolution are required simultaneously, it is possible to use a small diameter collimator followed by a scantube lens mechanism with a proper expansion ratio after the galvanometric mirrors to expand the OCT beam prior to the objective lens. Resonant scanning mirrors can achieve very high scanning rates ( $\sim 8 \mathrm{~K} \mathrm{~B}$-scans per second) in comparison with the galvanometric mirrors (200 B-scans per second for small regions). However, their lack of flexibility to scan desired patterns limits their application in OCT imaging. Moreover, in most SD-OCT applications, the imaging speed is limited by the detector line rate and, in practice, the speed of SD-OCT imaging is not limited by the speed of typical galvanometric scanning mirrors.

The physical dimensions of the mirrors dictate the separation between the two mirrors necessary to avoid collision. To optimally align the galvanometer, we need to place the back-focal plane of the objective in the middle point between the mirrors. In such an arrangement, none of the mirrors satisfies the telecentric configuration [65]. Telecentricity is important in OCT imaging and is discussed in the following section. The separation between the mirrors should be limited to the minimum possible distance; otherwise, it compromises the accuracy of OCT images. If the placement of the mirrors significantly reduces the telecentricity of the optical design, the OCT images suffer from a depth dependent magnification and the magnification is different along the $x$ and $y$ directions. When the separation is small, the imperfection is negligible. As the distance between the mirrors increases, this deformity also becomes noticeable and can introduce errors in measurements, especially when a high magnification objective lens is used. In cases when the mirrors cannot be placed sufficiently close to each other, this problem can be avoided by the use of relay optics between the mirrors. This relay mechanism projects the image of the first mirror ( $x$-axis) on the second mirror ( $y$-axis) $[66,67]$ and the second mirror is placed at the back-focal plane of the objective. Such a relay mechanism increases the size and cost of the OCT system. Another approach is to use twoaxis tilt mirrors $[68,69]$, which use a single mirror to scan along both $x$ and $y$ directions. Using current technologies, generally, galvanometric mirrors provide more linear and faster scanning speeds compared to two-axis tilt mirrors. The recent advances in microelectromechanical system (MEMS) devices have helped reduce this gap; however, currently, the speed of galvanometric mirrors outperforms that of two-axis tilt mirrors for most OCT applications.

The surface quality of galvanometric mirrors is important in low-coherence interferometry. The curvature of the mirrors' surface introduces phase-front distortion to the collimated beam which negatively impacts the image quality. The scanning mirrors should be stiff so that, during mechanical tension, at the time of large angular acceleration, the mirrors' 
surfaces remain flat. These considerations may affect the thickness (weight) and the material that are used in the construction process of the mirrors, which limit the speed and settling time of the galvanometric system. Therefore, when selecting the scanning mirrors, their speed as well as the mirror size and angular accuracy should match the desired parameters of the OCT system.

3.2.3. Objective Lens. Generally, in an OCT system, the lateral resolution and maximum possible field of view are limited by the objective lens. A high lateral resolution can be achieved using an objective that is designed to work at a large numerical aperture (NA). The lateral resolution of an OCT is defined as the radius of the beam spot at the focal point of the objective $[33,52]$. For a Gaussian beam of radius $r_{0}$ and wavelength $\lambda$ passing through a lens with the focal length of $f$, the spot size is inversely proportional to the NA [70]:

$$
\delta x=\frac{2 \lambda}{\pi} \frac{f}{r_{0}}=\frac{2 \lambda}{\pi N A} .
$$

Here, $\delta x$ is the theoretical lateral resolution. Increasing the NA increases the divergence angle of the Gaussian beam which translates to some reduction in the depth of focus. Depth of focus (DOF) of a Gaussian beam is the range in which the beam waist radius stays smaller than $\sqrt{2} \delta x$. Generally, DOF is considered as the range where the resolution is maintained close to the desired value, and outside this range the resolution drops quickly. For a Gaussian beam, the DOF is calculated by [70]

$$
b=\frac{\pi \delta x^{2}}{\lambda}=\frac{4 \lambda}{\pi N A^{2}} .
$$

Therefore, the DOF is directly proportional to the square of the spot size which poses a fundamental trade-off between the lateral resolution and the depth of focus. An optical setup with a low NA $(\sim 0.1)$ strikes a good balance between resolution and DOF for an OCT scanner in the infrared range of the spectrum. This is in contrast with many other microscopy technologies such as multiphoton fluorescence imaging, which demand a large NA to perform optical sectioning. For optical sectioning, a high numerical aperture is desirable since the beam outside the depth of focus diverges quickly and the effect of out-of-focus regions of a sample can be ignored. In OCT, low-coherence interferometry separates the photons that are backscattered at different depths in the tissue. Therefore, it is desirable for an OCT scanner to possess a uniform spot size across the depth of interest. This condition imposes a fundamental trade-off between the lateral resolution of an OCT scanner and its depth of focus.

Using a light source with shorter wavelengths, it is possible to strike a better balance between the lateral resolution and the depth of focus. However, a shorter wavelength experiences larger scattering in most biological tissues and has shorter penetration depth. In such samples, the scattering effect reduces the signal-to-noise ratio and ultimately the image quality. Therefore, depending on the application and the tissue properties, selecting a proper wavelength has a considerable impact on the quality and resolution of OCT images.

When a long central wavelength and a large depth of focus are necessary for an OCT application, it is recommended to use a Bessel beam. A Bessel beam with a spot size similar to a Gaussian beam offers a longer depth of focus [71-73]. The compromise here is that Bessel beams have relatively strong side lobes which can affect the accuracy of some OCT measurements. Also, implementing a system with a Bessel beam costs more due to the increase in the cost of some optical components.

Optical aberrations that are introduced by the objective lens in the sample arm can degrade the resolution of the imaging system. Low chromatic aberrations (longitudinal and lateral) are important when a light beam with a large bandwidth is used for OCT imaging. Chromatic aberrations prevent different wavelengths from coming into focus at the same focal point. Ultrawideband OCT light sources such as supercontinuum lasers are becoming more popular to achieve high axial resolution. If the objective lens is not designed for such a large bandwidth, chromatic aberrations prevent the scanner from achieving the theoretical lateral resolution. Proper coating of the objective for the desired wavelengths is also important to reduce the back reflection of the light from the objective. Since most objective lenses have a complex structure composed of several layers of different types of glass, the strength of the back reflection can be comparable to or stronger than the tissue backscattering.

One other important aberration in OCT imaging is astigmatism which may confine the area of the diffraction limited resolution to the center of the field of view. Astigmatism causes sagittal and tangential light rays to focus at different axial positions. Astigmatism reduces the lateral resolution of the OCT device when scanning the off-axis regions of a specimen. Besides the resolution, optical aberrations reduce the signal-to-noise ratio of OCT images. In a typical OCT device, the objective collects the photons that are backscattered from the tissue and sends them back toward the mirrors and the optical fiber. The fiber core acts as a pinhole and rejects the out-of-focus light. This significantly reduces the intensity of the light at the out-of-focus region significantly.

Other parameters that should be considered when selecting an OCT objective lens are magnification and telecentric property. Objective magnification determines the relationship between the change in the angle of the incident beam and the transversal displacement of the spot on the sample. A low magnification objective lens requires small mirror angles to cover a large field of view. The same scanning angles cover a smaller portion of the sample when a high magnification lens is used. For typical OCT imaging, low magnification objectives with large FOV are desirable. The design of most commercially available low magnification lenses does not allow the realization of small spot sizes, which is partially due to cost considerations. This couples the magnification of a lens to the imaging resolution which imposes a technical trade-off between the resolution and the field of view.

For laser scanning mechanisms, such as OCT, a linear relationship between the scanning angle and the transversal displacement allows the device to obtain a uniform sampling 
across the transverse plane by simply sweeping the angle of the galvanometric mirrors while the detector is recording at a constant rate. Such an objective is usually called an $f-\theta$ lens since the displacement of the beam is equal to the product of the effective focal length of the lens $(f)$ and the angle of incidence of the beam $(\theta)$. In general, $f-\theta$ lenses are able to produce a flat imaging plane and uniform resolution across the imaging plane. A non- $f-\theta$ lens realizes a nonlinear relationship between the mirror angle and the beam displacement on the sample. This effect can cause a nonuniform magnification across the transverse plane and distortion in the recorded OCT images. It is important to select an objective lens that introduces a low level of distortion. For OCT applications, the uniformity of magnification at various depths is desirable. For this purpose, the lens must be used in the telecentric configuration. That condition is achieved when the geometrical center between the mirrors is placed at the back-focal plane of the objective. A telecentric $f$ - $\theta$ lens provides a beam that is perpendicular to the flat imaging plane and its magnification is uniform within the depth of focus of the objective.

The abovementioned factors are related to the performance of an objective lens. Some other considerations are related to the mechanical properties of the objective. The distance between the back-focal plane and the surface of the lens is called back-focal length (BFL). The scanning mirrors should be placed such that the geometrical center of the mirrors collocates with the back-focal plane of the objective. If the BFL is sufficiently large, positioning the galvanometer will become more convenient. For objectives that are designed for high-resolution imaging, the BFL is short and it is almost impossible to place the mirrors in the designated space. In such conditions, a relay mechanism is necessary to image the mirrors on the desired positions $[65,67,74]$. Working distance in an objective is the distance between the focal plane and the surface of the lens. A large working distance provides more space between the sample and the lens. This is especially important for in vivo experiments where other devices and equipment are used simultaneously, for example, to collect other forms of data or to deliver certain chemicals to the organelle under study. In such conditions, a large working distance is very beneficial.

3.3. Beam Combination. For many applications, it is beneficial to integrate OCT imaging with other optical methodologies, such as fluorescence, white light, or laser speckle imaging. Such a combination can be achieved by placing a dichroic beam splitter in the path of the OCT beam in the sample arm. The dichroic mirror can be a long- or shortpass beam splitter; however, each additional component can introduce some imperfections or artifacts to OCT images. Most dichroic mirrors are designed to work with collimated beams. When a diverging or converging OCT beam passes through a slab of glass, different portions of the beam travel for different lengths inside the glass, which introduces optical aberrations that have a profound impact on the quality of images. Usually, this effect is more crucial when a long-pass mirror is used and the OCT beam passes through the dichroic glass.
3.4. Reference Arm. The reference arm in an OCT system replicates the light path of the sample arm. It may consist of the same components as the sample arm; however, it is possible to use dispersion-compensating (DC) blocks instead of some components, such as the objective lens, to reduce the cost. A DC component has dispersion properties similar to the original component. In the reference arm, a mirror is used in place of the sample. The path length of the reference arm should match the sample arm. In practice, it is desirable to place the reference mirror on a micromanipulator to adjust the reference arm length. In many applications, this length is adjusted such that the point of equal path length in the sample arm is placed a few hundred micrometers before the focal plane of the objective.

Since the reflection coefficient of a mirror is larger than a typical sample, the light that is collected back by the optical fiber in the reference arm is stronger than the collected light from the sample arm. A strong reference beam increases the signal-to-noise ratio (SNR) of OCT images. Also, a strong reference intensity reduces the visibility of the image artifacts caused by interference occurring between the reflected lights from different sample reflectors (see (5)). When imaging deep tissue or a weakly backscattering sample, a long exposure time is necessary to detect the weak backscattered light. In such cases, a strong reference beam saturates the detector and makes the reconstruction impossible. To adjust the intensity of the reference beam, one can place a variable attenuator or aperture in the reference arm. An alternative method of balancing the intensity of the reference beam, with respect to the sample beam, is to use a variable fiber coupler instead of the fixed ratio fiber couplers.

3.5. Spectrometer Arm. The photons that are collected in the sample and reference arms are combined by a fiber coupler in the spectrometer arm. If a 50:50 coupler is used, the sample and reference powers are equally divided between the output ports of the coupler and there is no preference between the ports. When a coupler with a ratio other than $50: 50$ is used to deliver more power to one of the arms, the same port that is connected to the light source should be used to detect the interference. In this condition, it is possible to use a single mode fiber optic circulator in the path of the light source to divert the path of the returned photons toward the detection (spectrometer) device.

The spectrometer arm usually consists of a single mode fiber, a collimating lens, a diffraction grating, a lens (or lens mechanism), and a line detector. The light that is emitted from the single mode fiber is collimated before illuminating the diffraction grating. The grating diffracts different wavelengths to different angles and the diffracted beam is imaged by a lens mechanism on the active area of a line CCD camera.

A line CCD camera is the detector in a SD-OCT setup. A combination of different design parameters including the source bandwidth, diffraction grating resolution, lens mechanism, and camera pixel size determines the axial imaging resolution and maximum depth that a SD-OCT can image which were partly discussed earlier in this paper. The overall bandwidth that is detected by the camera determines the axial resolution. A larger effective bandwidth results in 
a better axial resolution (see (7)). The maximum imaging depth depends on how finely the light spectrum is sampled by the camera. If the sampling is denser, using either a smaller detector pixel size or a lensing mechanism of longer focal length, the scanner provides a larger imaging depth; however, this mandates using a larger number of pixels to capture the same bandwidth to maintain the axial resolution. Besides the number and size of camera pixels and also the source bandwidth, the optical performance of the grating and imaging lens is important in the quality of the images. There are commercial spectrometers available which offer easy installation; however, it is not possible to adopt and tune them for every arbitrary light spectrum.

Here, we present a simple and fast guideline on how to select proper components and implement an efficient spectrometer for OCT applications.

3.5.1. Optical Grating. When selecting a grating, its designated wavelength, efficiency over the desired bandwidth, and polarization sensitivity should match the application. Transmission holographic gratings strike a good balance between efficiency, working spectrum, and insensitivity to minor misalignments, which is beneficial for OCT applications. Blazed gratings, in comparison with holographic gratings, offer a better efficiency around the working wavelength. However, their efficiency drops quickly for the wavelengths that are far from the central wavelength. This reduces the image signal-to-noise ratio (SNR) and/or degrades the OCT axial resolution by reducing the spectral bandwidth of the spectrometer.

For a light source with elliptical polarization, a grating with less polarization sensitivity is beneficial. When the efficiency is highly polarization dependent, the optical power of the diffracted light and the SNR of the generated images degrade. The grating needs to be aligned carefully so that the incident beam exposes the grating at the prescribed angle with respect to the grooving. Another advantage of transmission gratings is the lower sensitivity to the imperfections regarding the angle of incidence (AOI), compared to reflection gratings. However, to obtain the optimal performance, the alignment needs to provide the AOI that matches the grating specifications. Overall, transmission holographic gratings with small polarization preference meet the requirements of most OCT applications.

3.5.2. Lens Mechanism. The spectrometer lens mechanism focuses the light dispersed by the grating on a line detector which is placed on its focal plane. In this configuration, the spectrometer lens virtually acts as a scan lens. Different wavelengths exit the grating at different angles and are focused at different positions on a line along the direction of the light dispersion (Figure 3). Therefore, at the focal plane of the lens, different wavelengths are spatially separated and each pixel of the detector is receiving a portion of the spectrum based on its location. The lens FL should be selected such that the desired part of spectrum is projected on the extent of the sensor array. The bandwidth that is projected on the detector determines the theoretical axial resolution of the OCT system. If the FL is too large, only a portion of the bandwidth is received by the detector, which degrades the axial resolution. The SNR of OCT images reduces as well due to the power loss in the spectrometer. In this case, the spectrum is sampled more densely, which results in a larger imaging depth. In contrast, if the FL is too small, some of the camera pixels practically do not receive a sufficient number of photons and will not contribute to the reconstruction process. In such conditions, the axial resolution can still be calculated by (7) where $\Delta \lambda$ is the bandwidth of the light source. However, because of the larger sampling interval, the maximum imaging depth decreases (see (8)).

Since the pixel size is not infinitesimal, each pixel integrates over the portion of the spectrum that is projected on the sensor. Therefore, each detector's pixel acts as a lowpass filter which smoothens the interference patterns. In this process, the high frequency fringes are attenuated. Since the backscattered light from deeper objects creates higher frequency components in the interference pattern, the lowpass filtering results in a depth dependent attenuation [75]. This signal fall-off, which is sometimes called the sensitivity drop-off, causes the SNR of typical OCT images to drop quickly as depth increases.

Besides the physical dimension of the detector, the focal spot size of the lens mechanism is another factor that can cause signal fall-off. When the spectrometer spot size is smaller than the detector's pixel size, this effect can be ignored. When the spot size is comparable to or larger than the detector's pixel size, the energy of each wavelength spreads to more than one pixel which can be modeled by another low-pass filter being convolved with the interference signal, which worsens the signal fall-off. The spot size on the detector is directly proportional to the FL of the lens mechanism and inversely related to the collimated beam diameter (see (10)). If a lensing system with a large FL is required, then the size of the collimated beam that is incident on the grating should be selected accordingly to realize a small spot size on the detector. Optical aberrations such as astigmatism and Petzval field curvature result in a nonuniform spot size along the detector plane. When the aberrations are significant, the varying spot size causes an attenuation in the interference pattern, which varies as a function of the position across the detector. Therefore, these aberrations generate wavelength dependent attenuation which acts as a spectral filter that may degrade the axial resolution of the system as depth increases. Using a proper lens mechanism and collimating lens, it is possible to produce a sufficiently small spot size on the detector and reduce optical aberrations to achieve a high axial resolution and minimize the signal fall-off.

3.5.3. Detector. In recent years, several line CCD cameras were designed and manufactured particularly for SD-OCT imaging. Their spectral response, dark noise, exposure time, data transfer rate, and pixel size allow for fast, highresolution, high-dynamic-range, and high-sensitivity OCT imaging.

The sensitivity of an OCT scanner is defined as the inverse of the sample reflectivity which produces an amplitude equal to the noise amplitude in the OCT image. This value has a great impact on the image quality and is affected by several 
parameters in the detector. The dynamic range is defined as the ratio between the minimum reflectivity that causes saturation and the noise level in the detector. Therefore, this value represents the span of reflectivity values in the sample arm that can be detected by the scanner. Practically, the dynamic range of a system is smaller than its sensitivity. The typical values for sensitivity and dynamic range are $\sim 100 \mathrm{~dB}$ and $\sim 60 \mathrm{~dB}$, respectively [33]. The detector array records the reference and sample signals along with the interference signal. Generally, the amplitude of the reference signal is much stronger than of the interference signal; therefore, the detector should have a large photoelectron capacity to record weak interference signals that are superimposed on top of the strong reference signal. Shot noise, readout noise, thermal noise, and analog-to-digital conversion noise are the typical forms of noise that limit the sensitivity of an OCT system. It is desirable for a detector to work at the shot noise limit which is a fundamental noise in imaging techniques. A small receiver noise is essential for a SD-OCT detector to take advantage of its large photoelectron capacity and to achieve a shot noise-limited performance [37, 40, 41]. Since in SDOCT imaging the depth profile is calculated using a discrete Fourier transform, to achieve a dynamic range of $60 \mathrm{~dB}$ in the image, it is not required for the detector to capture the interference signal with such a large dynamic range. A detector with a 12-bit analog-to-digital converter provides a sufficient bit depth to achieve a sufficient dynamic range and low discretization noise for SD-OCT imaging.

The scanning speed of a SD-OCT imaging system is mainly limited by the line rate of its detector. Commercially available line CCD cameras are able to provide a scan rate of $>240 \mathrm{k}$ lines per second at the cost of larger readout noise and a smaller dynamic range compared to lower speeds. Although such detectors are sufficiently fast for many applications, faster and more sensitive detectors are beneficial in certain in vivo experiments, for example, to reduce motion artifacts or perform Doppler velocimetry of fast moving particles. By employing the new state-of-the-art electron-injection based detectors [76], it is possible to improve the sensitivity and dynamic range of the OCT imaging systems at higher imaging speeds.

The spectral response of the detector acts as a filter, similar to other optical components. If the spectral response is smaller than the light source such that it deforms the spectrum of the recorded signal in comparison with the source, then it can degrade the axial resolution of the OCT imaging system. Most line detectors have fairly flat response over a large spectral range and one can ignore their effect on the axial resolution. However, to obtain an ultrahigh resolution, the spectral response of the detector should match the spectrum of the light source.

In diffraction-based spectrometers, each wavelength is diffracted to a different direction. The angle between the diffracted light beam and the optical axis of the grating is not proportional to the light frequency ( $\kappa$ space) [36]. Consequently, the line CCD nonuniformly samples the interference pattern in the $\kappa$ space. Applying the Fourier transform (see (6)) to this sampled interference signal to obtain the depth information prior to any further processing results in low resolution images and the reconstructed image suffers from a low signal-to-noise ratio (SNR). As a result, the majority of spectral-domain OCT systems apply a resampling routine as part of the OCT signal processing procedures to convert a signal that is recorded in the $\lambda$ space to a signal in the $\kappa$ space. However, some hardware solution has been offered by different research groups to avoid the resampling process. For example, $\mathrm{Hu}$ et al. [64] and Gelikonov et al. [77] have used a custom-designed prism between the grating and the lens in their interferometer to compensate for this nonuniform frequency sampling property of the grating. However, this method involves the custom design of a specific prism. Placing the prism in the spectrometer to obtain the uniform sampling in the frequency domain is a very sensitive and time-consuming task. Moreover, there are some imperfections, like the mismatch between the dispersion in the sample and reference arms, which eventually demands a software-based resampling process and cannot be solved readily by placing a prism in the spectrometer arm. Therefore, a software-based process would be essential to obtain a uniformly sampled signal in the frequency domain [75]. The majority of spectral-domain OCT systems perform the resampling as part of the OCT signal processing procedures.

3.6. Fibers, Coupler, and Circulator. For typical fiber-based OCT applications, single mode optical fibers and single mode fiber-based components are required. The core diameter of such fibers is in the range of a few microns to $10 \mu \mathrm{m}$ depending on the central wavelength. The designed spectral bandwidth of the fibers should match the light source bandwidth. Otherwise, the coupling efficiency might be low or the fiber may act as a multimode fiber. Although there are some studies that demonstrate the benefit of using multimode fibers in certain applications [78-81], in a typical OCT design, using a multimode fiber can cause numerous artifacts in the image.

The fibers should also provide angled physical contact (APC) with the rest of the system to avoid image artifacts. Noticeable back reflection of light from any object can contaminate OCT images. The back reflection from the surface of the fiber tip is sufficiently strong to cause image artifacts. For this reason, the fibers need to be polished to have an angled surface which reduces the power of the back-reflected light that is coupled back to the fiber. When using single mode fibers with angled polish, other fiberbased components (collimators, fiber coupler, light source, and optical fiber circulator) need to be selected accordingly. The components that are compatible with angled polish fibers are declared by "APC" in their naming typically.

Light polarization may change when it passes through a typical single mode fiber. If the sample polarization and reference polarization are different, this potentially reduces the interference amplitude between them and reduces the SNR of OCT images. To achieve high SNR imaging, it is recommended to use polarization controllers in sample, reference, and spectrometer arms. This allows the user to adjust the polarization of the sample and reference arms to achieve a high imaging SNR. However, for most biological samples, this step is not necessary to obtain OCT images. Polarization 


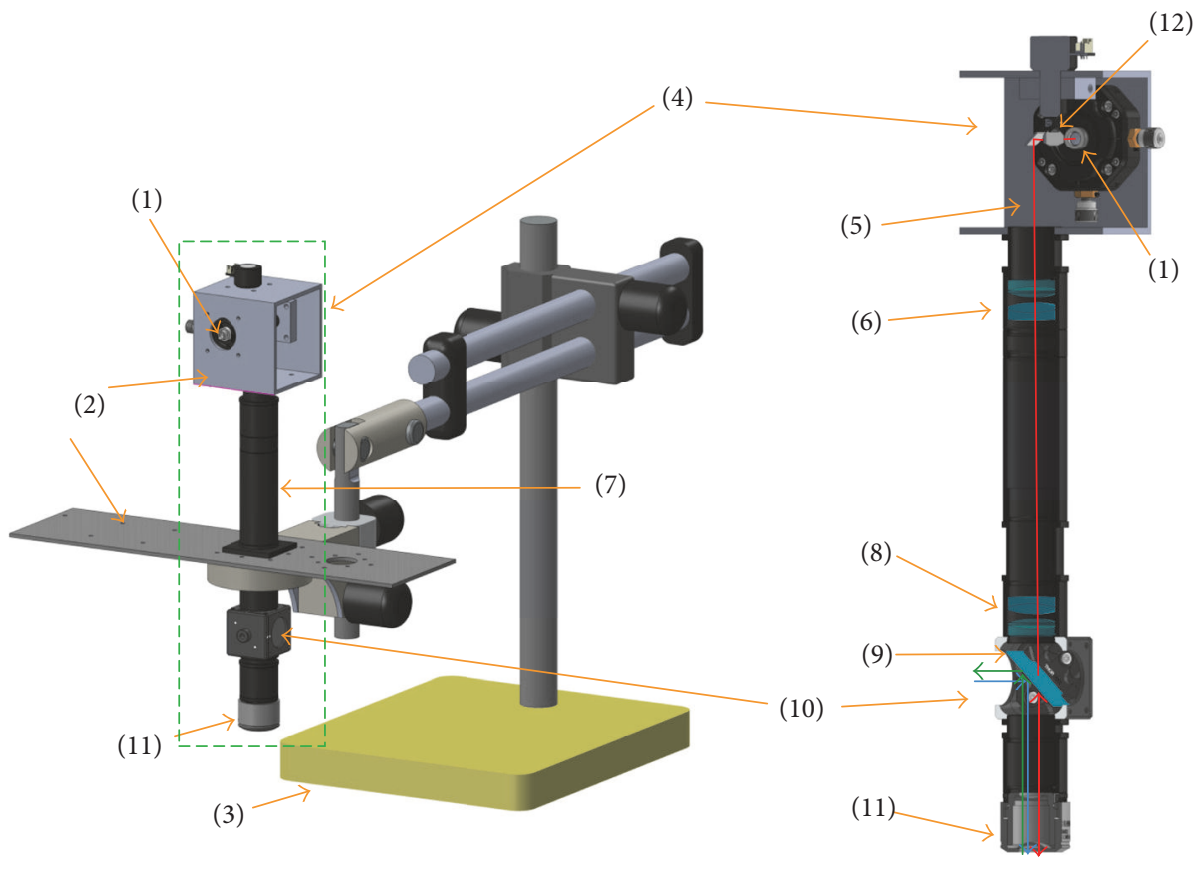

(a)

(b)

FIGURE 2: Sample arm. This image demonstrates the design of the sample arm that is mounted on a typical microscope stand. This system uses two identical semi-Plössl lenses as scan and tube lenses. The cross section shows the optics including galvanometric mirrors, scan and tube lens mechanisms, and the infrared dichroic mirror. (1) OCT collimator, (2) custom mounts, (3) commercial microscope stand, (4) scan head, (5) OCT beam, (6) scan lens, (7) lens tube, (8) tube lens, (9) dichroic mirror, (10) visible port, (11) objective lens, and (12) galvanometric mirrors. The SOLIDWORKS designs of lens tubes and cage cube-mounted beam splitters are adapted from Thorlabs (Thorlabs, NJ, USA). The microscope stand model has been modified from [45].

maintaining fibers should be used for polarization-sensitive OCT (PS-OCT) imaging. These fibers are designed to not alter the light polarization and are suitable for PS-OCT applications.

\section{Materials and Methods: OCT Scanner Development}

In this section, we discuss details of a SD-OCT system that we implemented for brain imaging applications. With slight modifications, the same design can be used for other applications that may require a different resolution, imaging depth, or light spectrum.

4.1. Light Source. To image up to several hundred micrometers deep inside a scattering medium, such as the brain tissue, with axial and lateral resolutions of $\sim 5 \mu \mathrm{m}$ or better, a light source with a long central wavelength is preferred. According to (7), the axial resolution of a SD-OCT system is linearly proportional to the square of central wavelength of the light source and inversely proportional to its spectral bandwidth. To achieve a balance between the penetration depth and resolution, a light source with a central wavelength of $1300 \mathrm{~nm}$ and FWHM bandwidth of $>170 \mathrm{~nm}$ (LS2000B, Thorlabs, NJ, USA) was used. With this light source, the theoretical axial resolution is about $4.4 \mu \mathrm{m}$ in air, which meets our requirement.
Since the infrared light is not visible to the human eye, for alignment purposes, an aiming red laser was utilized in this design. The beam of this laser is coupled to the infrared fiber path via a fiber coupler. The same fiber coupler splits the OCT light beam between the reference and the sample arms.

4.2. Sample Arm. Our goal was to obtain a lateral resolution of $5 \mu \mathrm{m}$, so we accordingly selected an OCT objective lens (LSM02, Thorlabs, NJ, USA) with an effective focal length of $18 \mathrm{~mm}$. In combination with an incident beam diameter of $3.4 \mathrm{~mm}$, this lens provides a theoretical FWHM spot size of $\sim 5.2 \mu \mathrm{m}$. The collimated beam was produced by connecting a single mode optical fiber to a collimating package with the effective focal length of $18.86 \mathrm{~mm}$ (F280APC-C, Thorlabs, NJ, USA).

Our sample arm uses a set of galvanometric mirrors (GMs) to raster-scan the tissue. The GMs are designed for beam diameters smaller than $5 \mathrm{~mm}$ (GVSM002, Thorlabs, NJ, USA). The geometrical middle point between the mirrors should be positioned at the back-focal plane of the objective lens (OL). In this OL, as in most objective lens compounds, the back-focal plane is very close to the objective, which makes it challenging to place the mirrors at the designated positions. We installed the mirrors on a custom-made holder (Figure 2), with separation of $\sim 10 \mathrm{~mm}$ between the mirrors to avoid accidental collision. A scan-tube 


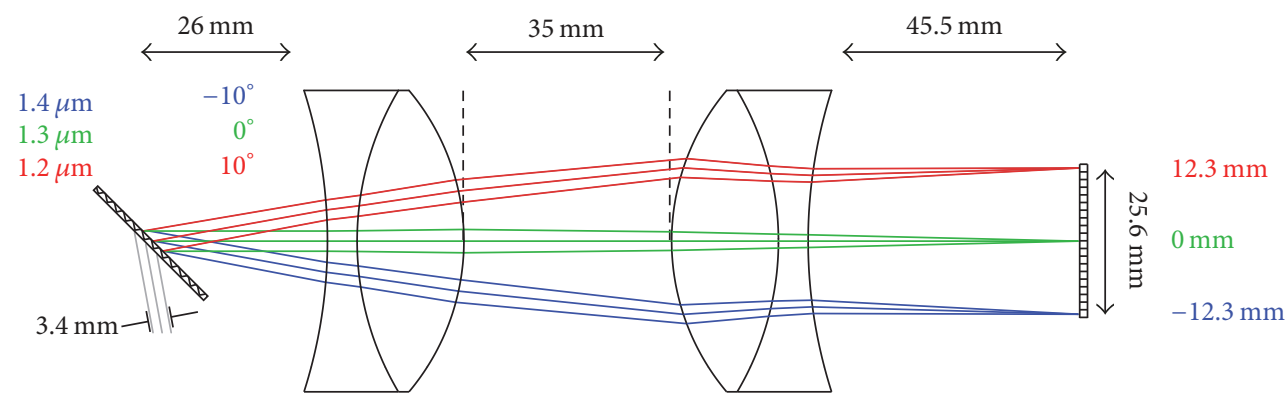

(a)
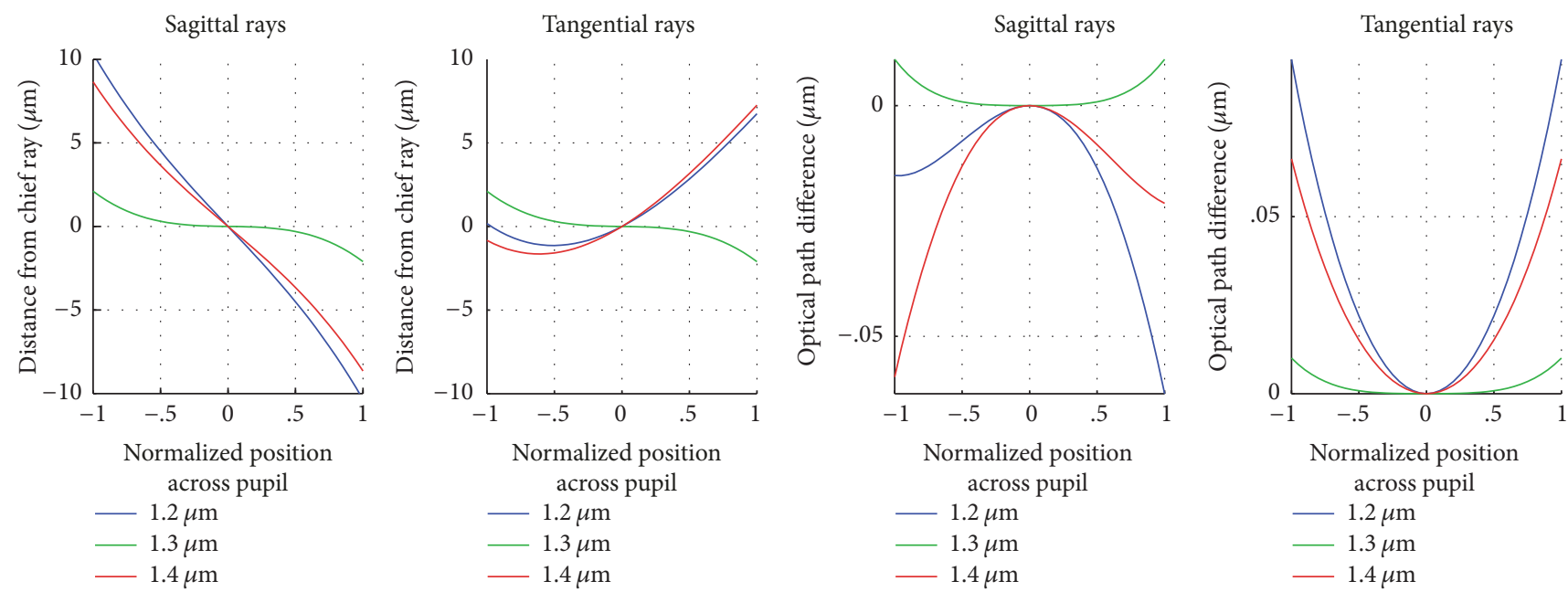

(b)
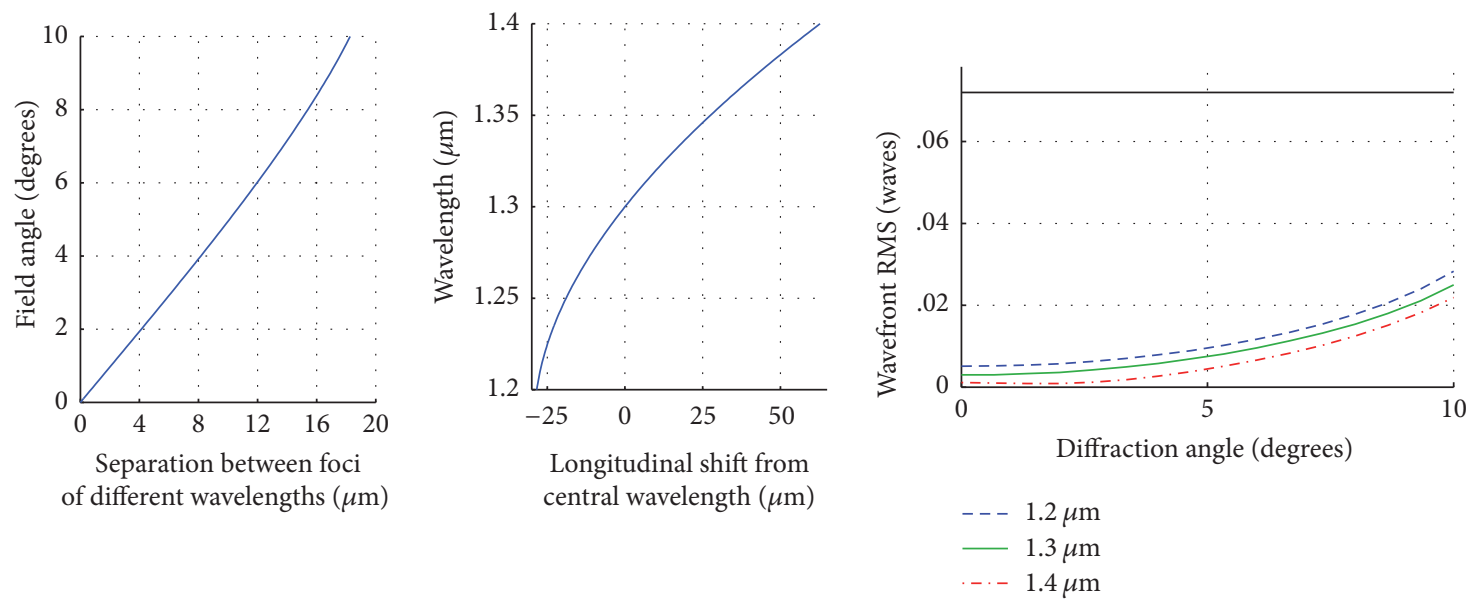

(c)

(d)

Figure 3: A schematic of the spectrometer. The grating diffracts the broadband collimated beam. The lens system acts as a scan lens and focuses the diffracted light beams on the focal plane array of a line scan camera. The focal length of the lens mechanism is designed such that the desired spectrum ( $1200 \mathrm{~nm}$ to $1400 \mathrm{~nm}$ ) is covering the extent of the detector array. Optical aberrations in this scan lens system are minimized to reduce the sensitivity roll-off of the OCT images.

lens mechanism images the middle point between the mirrors to the back-focal plane of the objective [74]. With the use of the scan-tube lens, there is space to place a dichroic beam splitter in the sample arm. This dichroic beam splitter can separate OCT and visible light paths (visible port in Figure 2) to allow simultaneous OCT and bright-field or fluorescence imaging. The current design of the system is for a longpass dichroic beam splitter; however, a custom mount can be slightly modified to accommodate a short-pass dichroic mirror instead. When using a long-pass dichroic, the OCT 


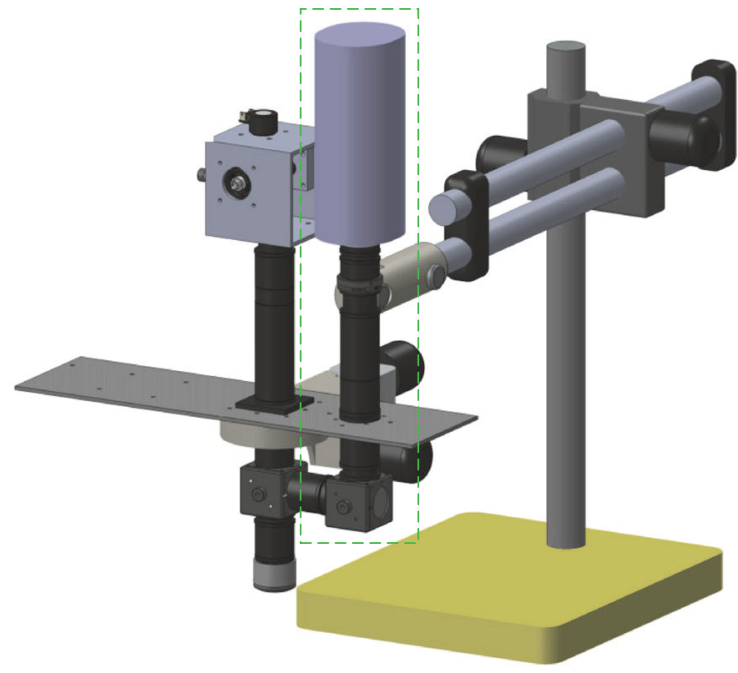

(a)

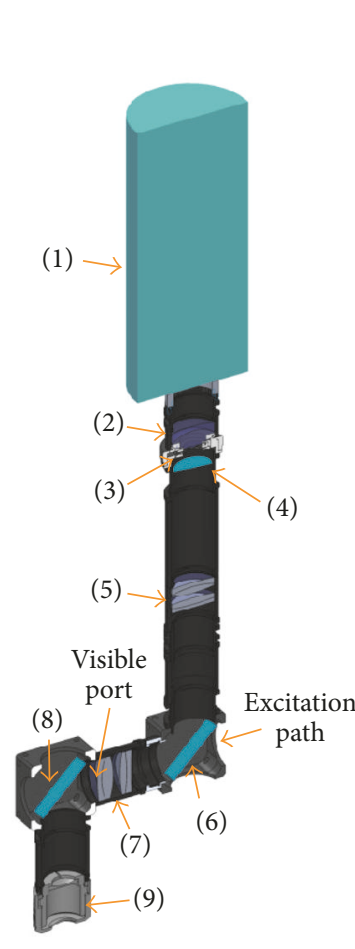

(b)

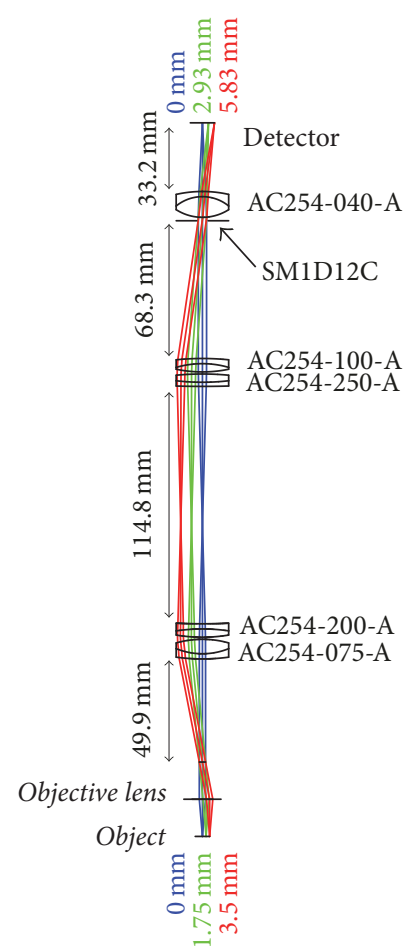

(c)

FIGURE 4: An example of fluorescence imaging integrated with the OCT sample arm. The system uses the same objectives for OCT and fluorescence imaging for simultaneous OCT and fluorescence recordings. (a) The placement of fluorescence imaging path in the OCT sample arm. (b) The SOLIDWORKS design of the fluorescence imaging path. (c) The schematic of the optical design of the path. The fluorescence imaging path uses two semi-Plössl lenses to image the back-focal plane of the objective to the entrance pupil of an imaging lens. There is an adjustable iris in this path to adjust the aperture diameter of the imaging system. This is helpful to reduce the aberration in the optical path and improve the imaging resolution. (1) Fluorescence camera (EXi Aqua, QImaging, BC, Canada), (2) adjustable iris (SM1D12CZ, Thorlabs, NJ, USA), (3) imaging lens (AC254-040-A, Thorlabs, NJ, USA), (4) optical filter, (5) semi-Plössl lens (AC254-100-A + AC254-250-A), (6) visible light dichroic mirror (FF440-520-Di01, Semrock Inc., NY, USA), (7) semi-Plössl lens (AC254-075-A + AC254-200-A), (8) long-pass infrared dichroic mirror (FF875-Di01, Semrock Inc., NY, USA), and (9) objective lens.

beam passes through the glass slab. The length that light travels via the glass varies as a function of each scanning angle. This causes a spurious change in the depth of reconstructed OCT images across the transversal plane as the beam raster-scans the tissue. This spurious change in the depth may appear as a bias in OCT Doppler velocity measurements. A short-pass dichroic beam splitter does not introduce such artifacts; however, multiple reflections from different layers of reflectors in a short-pass filter can cause strong artifacts in the OCT image that appear as replications of the original image. A short-pass dichroic mirror should be selected carefully to avoid such artifacts. We used a long-pass beam splitter (FF875-Di01, Semrock Inc., NY, USA). This dichroic mirror reflects wavelengths that are shorter than $900 \mathrm{~nm}$. A simple design of a fluorescence/bright-field imaging path is shown in Figure 4. To avoid bias in Doppler measurements, when using a long-pass dichroic mirror, we suggest using a bidirectional scanning mechanism. In the bidirectional scanning mechanism, the same cross section is scanned from two opposite directions, and after the Doppler calculations, the measurements are averaged. The Doppler artifacts that are introduced by structural imperfections, like the dichroic glass, are additively negative in the two recordings and are canceled out.

The sample arm (including the fluorescence imaging) is mounted on a custom-made adapter and a typical microscope double-arm boom stand. This allows for adjustment of the OCT head angle to approach the specimen from the desired angle.

4.3. Visible Path. The design of the system allows for installation of a visible path on the imaging device. The visible path can be used for delivery of optogenetic stimulation light pulses or incorporating a secondary imaging mechanism such as optical intrinsic signal imaging (OICI) or fluorescence imaging. In this part, we offer a sample fluorescence imaging design that has been added and tested on this device. Figure 4 represents the optical design and hardware realization of the imaging path.

4.4. Reference Arm. Ideally, in an OCT system, the reference arm should replicate the length that light travels and the dispersion that it experiences in the sample arm. If the light dispersion in the sample arm is not properly replicated in 


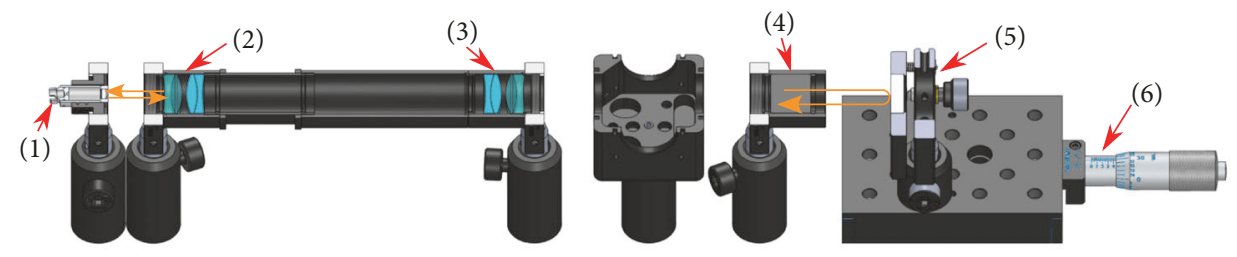

(a)

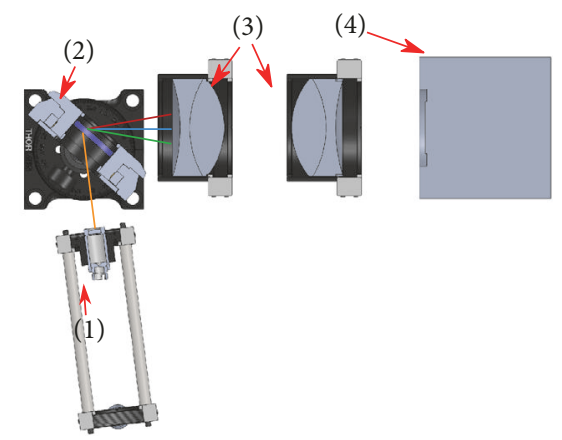

(b)

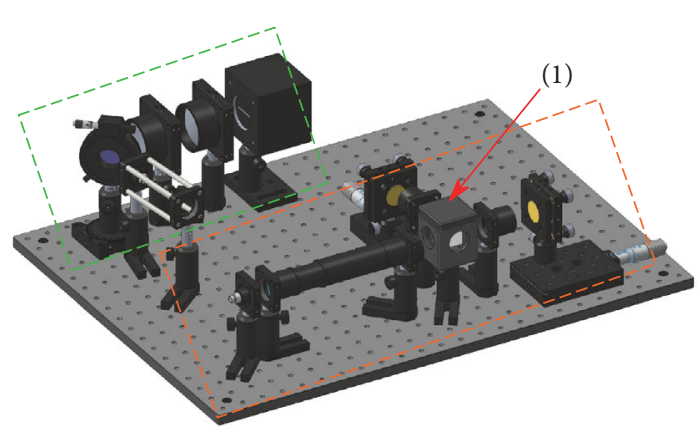

(c)

FIGURE 5: SOLIDWORKS design of the reference and spectrometer arms. (a) The CAD design of the reference arm. (1) Fiber connector, (2) scan lens, (3) tube lens, (4) dispersion compensator for the objective, (5) a gold mirror installed on a kinematic mount with adjustable angles, and (6) translational stage to precisely control the position of the reference mirror. (b) The CAD design of the spectrometer arm. The grating has an optimal incident angle of $48.6^{\circ}$ and diffraction angle of $48.6^{\circ}$. It is mounted on rotatory mounts to adjust the angles of the grating with respect to the OCT beam and the lens mechanism. (1) Fiber connection, (2) grating installed on rotatory mounts, (3) Plössl lens, and (4) line CCD camera. (c) The reference and spectrometer arms are installed on an optical breadboard to facilitate transportation. There is a movable mirror mount, component (1), in the reference arm that can change the beam path to match different objective lenses in the sample arm. The reference and spectrometer arms are encircled with orange and green rectangles, respectively.

the reference arm, it may greatly affect the axial resolution and quality of the OCT images. To minimize the dispersion mismatch between sample and reference arms, we assembled the reference arm with identical components which were used in our sample arm, except the scan head and the objective lens. In the reference arm, we used a single glass dispersion compensator (DC) (LSM02DC, Thorlabs, NJ, USA) to replicate the optical path of the beam inside the objective lens (Figure 5). This design reduces the cost. At the end of our reference arm, we use a protected gold mirror (PF10-03-M01, Thorlabs, NJ, USA) to obtain a flat reflection spectrum within the wavelength range of our light source. The light power that is back-reflected from a mirror is typically much stronger than the backscattered light from tissue in the sample arm. We installed an adjustable iris in the reference arm to tune the reference power. A removable right-angle mirror is used in the reference arm to incorporate multiple optical paths in the reference arm. This is useful when more than one objective lens mechanism is used during an experiment.

4.5. Spectrometer. To record interference patterns, we used a custom-made spectrometer which consists of a transmission grating, a Plössl compound lens, and a line charge-coupled device (L-CCD). We chose a volume phase holographic transmission grating (Wasatch Photonics, UT, USA) with 1145 grooves per $\mathrm{mm}$ to obtain a large bandwidth and a balanced performance for different light polarization. The optimal incident and diffraction angles at $\lambda=1.31 \mu \mathrm{m}$ for this grating are both $\sim 48.6^{\circ}$. These requirements were accommodated by installing the grating on two rotation mounts (PRM1 and RP01, Thorlabs, NJ, USA) for the fine adjustment of yaw and roll angles (Figure 5). The beam was delivered to the spectrometer via an SMF-28e+ single mode fiber (CIR1310-50-APC, Thorlabs, NJ, USA) and a collimating package (F280APC-C, Thorlabs, NJ, USA).

The diffracted beam is imaged on the L-CCD by two achromatic doublet lenses (AC508-150-C, Thorlabs, NJ, USA) which form a Plössl compound (Figure 3) with an effective focal length of $\sim 72 \mathrm{~mm}$. The L-CCD detector in this system (SU1024-LDH2, Sensors Unlimited, NJ, USA) has 1024 pixels with a $25 \mu \mathrm{m}$ pixel pitch and aperture height of $500 \mu \mathrm{m}$. The length of the focal plane array in this camera is $25.6 \mathrm{~mm}$ which is sufficient to receive at least $200 \mathrm{~nm}$ of the spectrum $(1200 \mathrm{~nm}$ to $1400 \mathrm{~nm})$ in combination with our lensing mechanism. For an incident beam diameter of $3.4 \mathrm{~mm}$, this lens compound forms a theoretical FWHM spot size of $\sim 20 \mu \mathrm{m}$ which is smaller than the camera pixel pitch. Optical aberrations that are introduced by this lens system are sufficiently small (Figures 3(b) and 3(c)) and the root-meansquare wavefront error stays below the diffraction limit at different diffraction angles (Figure 3(d)). The small spot size reduces the sensitivity roll-off by reducing the spread of each wavelength over multiple pixels. 
4.6. Fiber-Based Components. The light source was directly connected to a fiber optic circulator (CIR-1310-50-APC, Thorlabs, NJ, USA). The circulator illuminates a 50/50 fiber coupler (FC1310-70-50-APC, Thorlabs, NJ, USA) which splits the OCT beam between the sample and reference arms via two single mode fibers with APC cuts (P3-980A-FC2, Thorlabs, NJ, USA). The backscattered or back-reflected light for the sample and reference arms is recombined by the fiber coupler and guided toward the spectrometer arm via the fiber circulator. The fiber coupler possesses two input ports where one is used for OCT beam and the other is connected to a red laser diode. The red light is used for alignment purposes and to help the operator guide the beam to cover the region of interest during OCT imaging. When no visible light for alignment is used and a fiber coupler with 50/50 split ratio is being used, no fiber circulator is needed as the free coupler port can be connected to the spectrometer directly.

4.7. Animal Preparation. We used OCT to visualize the vascular network in the cerebral cortex of Sprague-Dawley rats. All animal procedures were approved by the Institutional Animal Care and Use Committee (IACUC) at the University of Wisconsin and Medical College of Wisconsin. During all surgical and imaging procedures, the animals were anesthetized using isoflurane and body temperature was regulated using a heated water pad. For intravital imaging of the cerebrovasculature, a closed cranial window of $\sim 5 \mathrm{~mm}$ diameter was prepared. Briefly, a craniotomy was made and the dura mater covering the brain within the window was removed to enhance resolution and depth of imaging. Prior to imaging, the craniotomy was sealed with a glass coverslip.

\section{Results}

By placing a gold mirror (PF10-03-M01, Thorlabs, NJ, USA) in the sample arm, we measured the axial resolution of this device. This was done by imaging the mirror and then measuring the FWHM of the recorded A-scans along the axial direction. The mirror surface acts as a Dirac delta input to the imaging system along the depth direction; however, due to the finite axial resolution of the system, we observed that the Ascan broadens along the depth (Figure 6). The practical axial resolution of the system is $\sim 5.6 \mu \mathrm{m}$, which is slightly larger than the theoretical resolution. The imperfections in the components and optical aberrations in lensing mechanisms can contribute to the degradation of axial resolution. The non-Gaussian distribution of the source power is another important factor that negatively affects the resolution.

Using a 3D phantom with embedded subresolution gold nanoshells [74], we measured the point spread function (PSF) of this OCT scanner at its focal plane. The results verify a lateral full width at half maximum (FWHM) spot size of $\sim 5.6 \mu \mathrm{m}$ and $\sim 6.4 \mu \mathrm{m}$ (or equally $1 / e^{2}$ spot diameters of $9.5 \mu \mathrm{m}$ and $10.9 \mu \mathrm{m}$ ) along the fast and slow galvanometric axes, respectively. The lateral resolution was consistent across the field of view of $4 \mathrm{~mm}$ in diameter (Figure 7). These OCT resolution measurements are in close agreement with the empirical spot size measurements provided by the objective

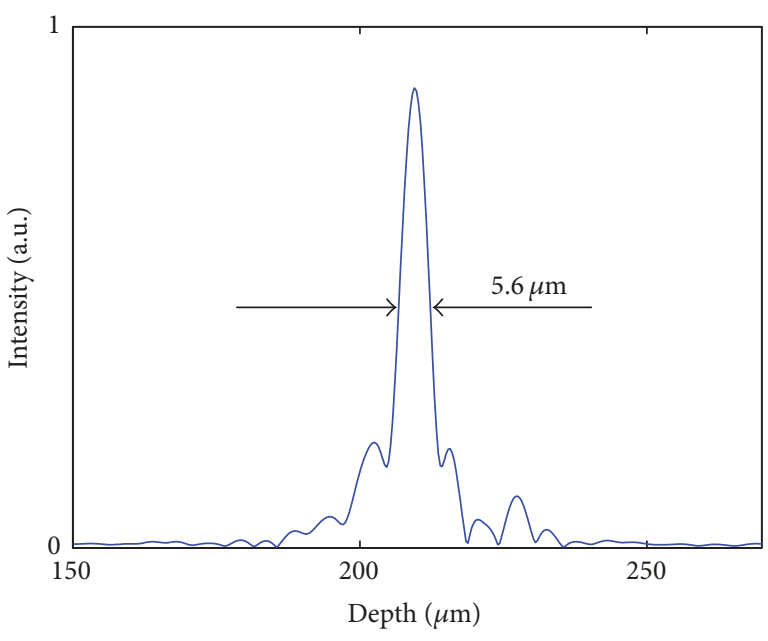

FIGURE 6: The measured axial resolution of the implemented SDOCT system.

vendor. When used with an incident beam diameter of $4 \mathrm{~mm}$, they measured a $1 / e^{2}$ spot size of $\sim 11 \mu \mathrm{m}$ at the focal point of the objective.

During our resolution measurements, the focal plane of the image was set at $\sim 390 \mu \mathrm{m}$ below the surface of the phantom to allow for recording the PSF of the OCT scanner at different distances from the focal plane. At the focal plane, we observed a quasi-Gaussian and localized distribution of the intensity (Figure 8). At deeper regions, the spread of the Gaussian increases and its intensity drops. At regions above the focal plane, we see that the spot size shrinks while a ring starts to form around it. This ring is visible for particles that are located $\sim 100 \mu \mathrm{m}$ above the focal plane. At shallower regions (e.g., $120 \mu \mathrm{m}$ above the focal plane), the ring becomes almost as intense as the central spot. We anticipate that the combination of spherical aberrations in the objective and the beam cut at the collimating packages are contributing to the formation of the ring which appears in our PSF measurements. To investigate the effect of tube/scan lens mechanism on the formation of the ring, we conducted similar measurements by modifying the design of our sample arm to place the objective lens directly after the scanning mirrors. When the scan/tube lens mechanism was removed from the OCT path, our measurements (not included in this manuscript) revealed a similar ring pattern above the focal plane of the OCT images. This observation confirmed that the intermediary lens mechanism does not significantly contribute to the formation of the surrounding rings in the OCT PSF.

The intensity change in the OCT images across the field of view was measured using the $3 \mathrm{D}$ phantom. At each desired location in the field of view, a $400 \mu \mathrm{m} \times 400 \mu \mathrm{m}$ block of the phantom was imaged and the average intensity of the detected nanoshells at the focal plane of the system was calculated. This calculation was repeated at different locations across the field of view and results are presented in Figure 9. The system is able to provide almost a flat intensity (less than $2 \mathrm{~dB}$ drop in the intensity) for a field of view as wide as $4 \mathrm{~mm}$ in diameter. However, when approaching the boundary of the diffraction limited field of view $(\sim 4.7 \mathrm{~mm})$, the intensity 


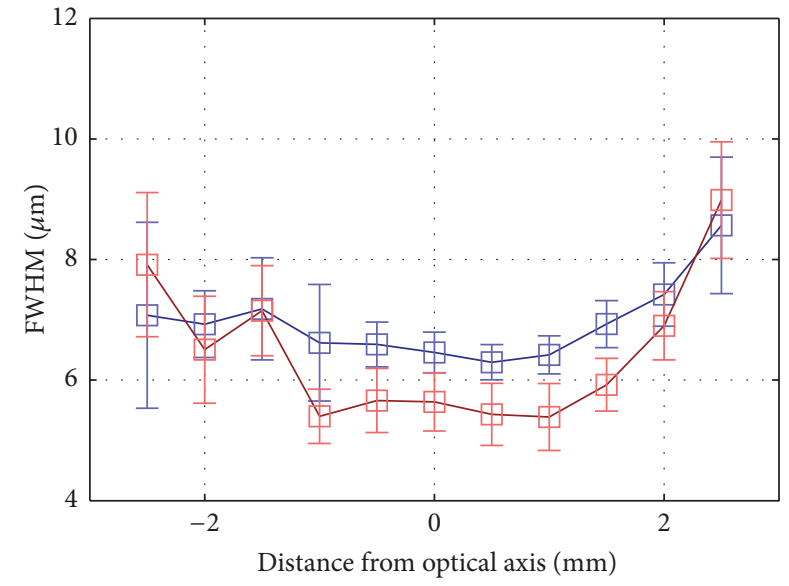

- Resolution along fast axis

— Resolution along slow axis

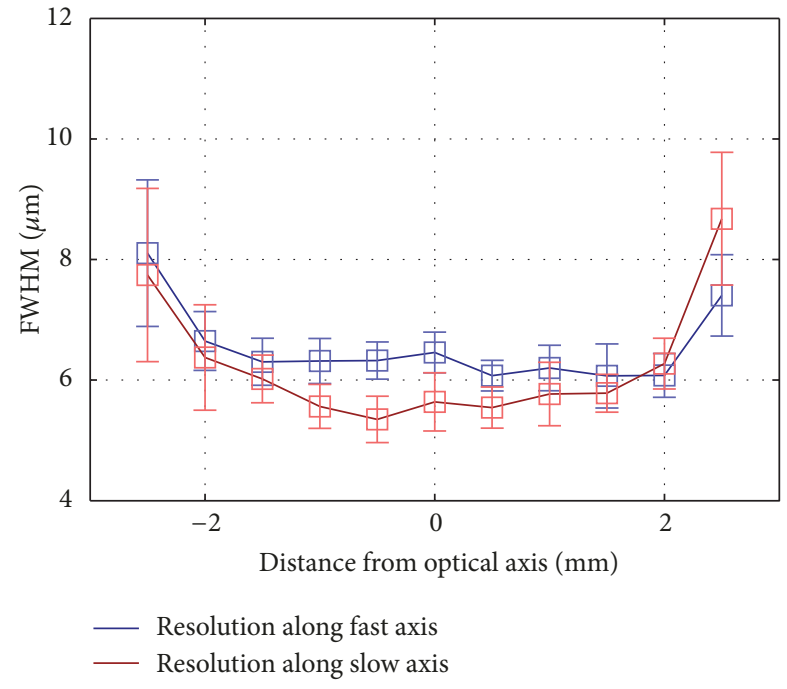

FIGURE 7: Variation in the lateral resolution of the implemented SD-OCT. Within the radius of $2 \mathrm{~mm}$, the FWHM resolution stays below $7.1 \mu \mathrm{m}$. The maximum diffraction limited field of view for this objective is $\sim 4.7 \mathrm{~mm}$ in diameter. When approaching this limit, the FWHM increases to $\sim 8 \mu \mathrm{m}$. The error bars mark the standard deviations. There are at least 70 measurements at each location.

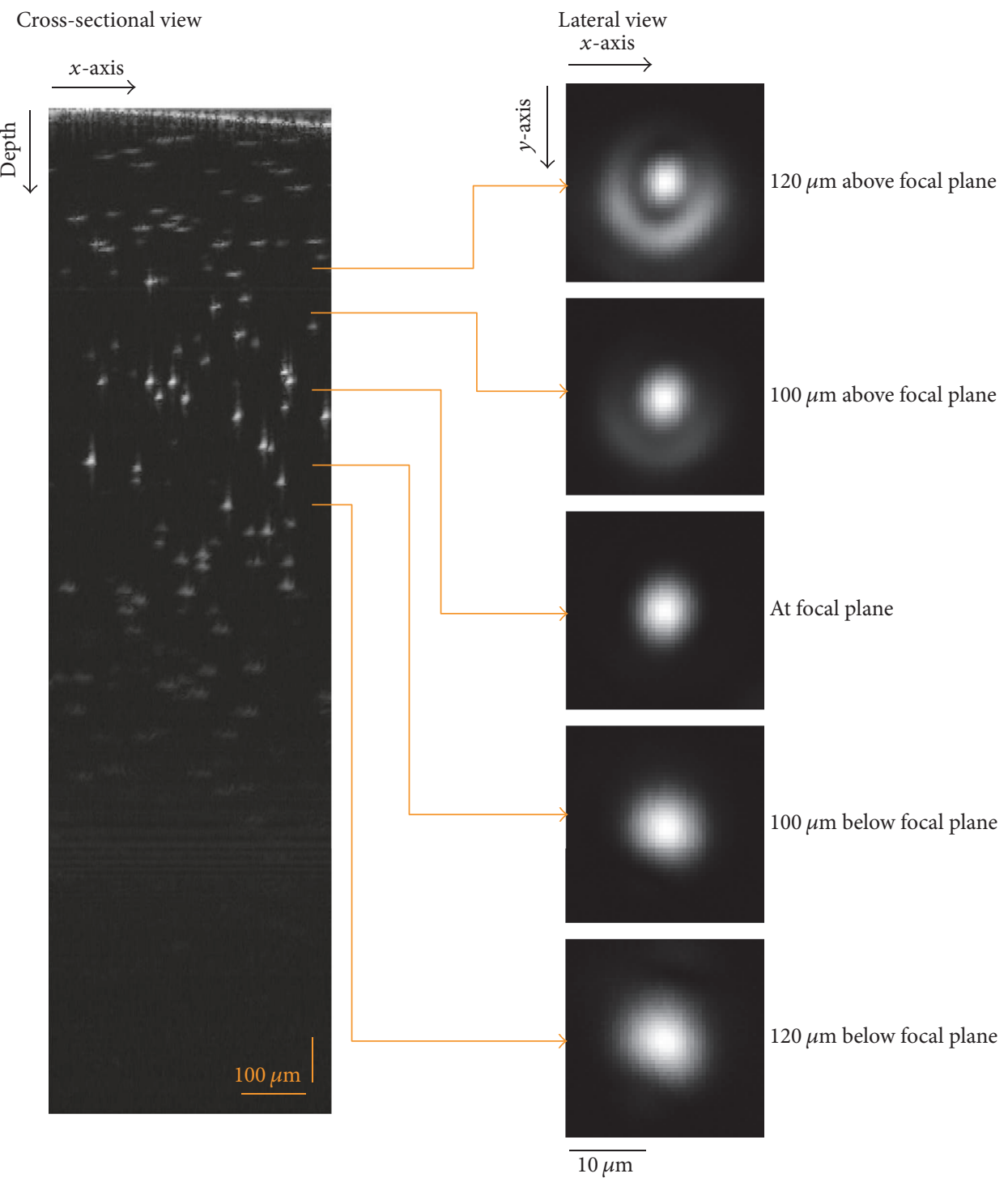

FIGURE 8: The lateral point spread function (PSF) of the custom-made OCT scanner. The PSF was measured using a gold nanoshell embedded phantom when the focal plane was placed $\sim 390 \mu \mathrm{m}$ below the surface of the phantom. The lateral PSF includes some rings at regions above the focal plane. 

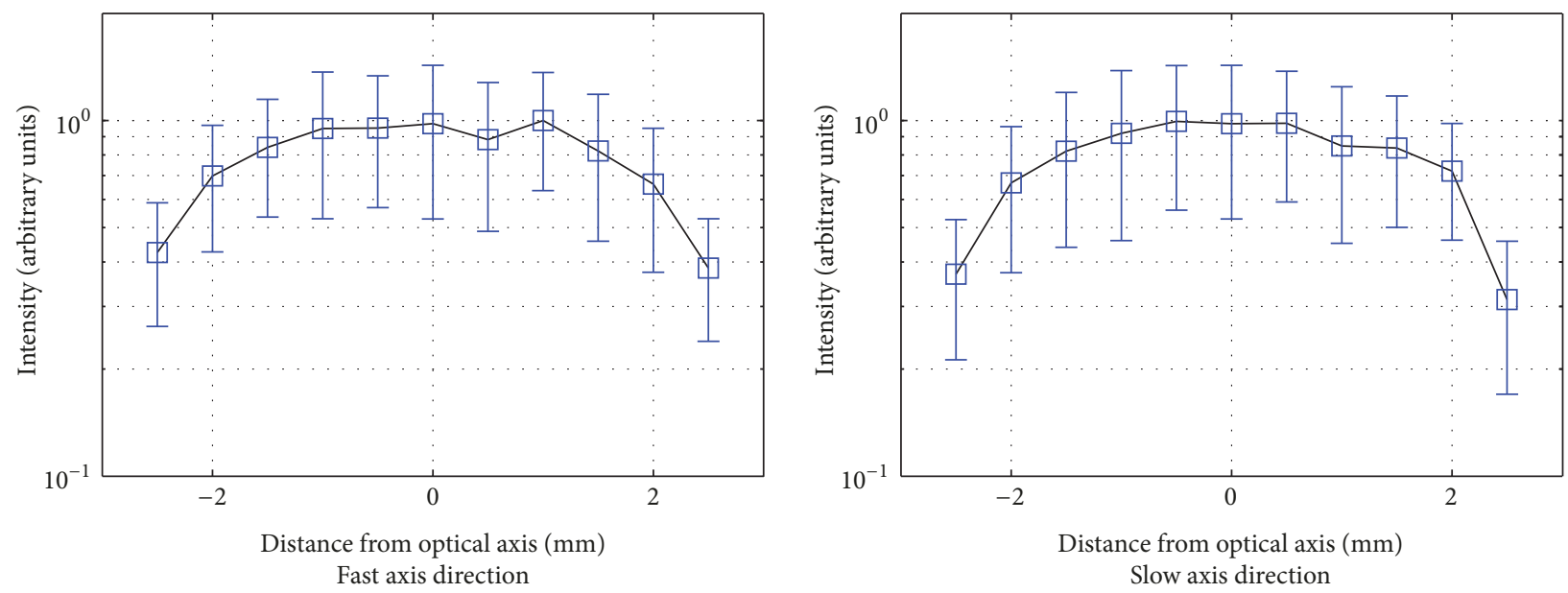

Figure 9: Variation in the intensity of OCT images across the field of view. At the borders of our field of view ( $4.7 \mathrm{~mm}$ in diameter), the intensity drops rapidly.
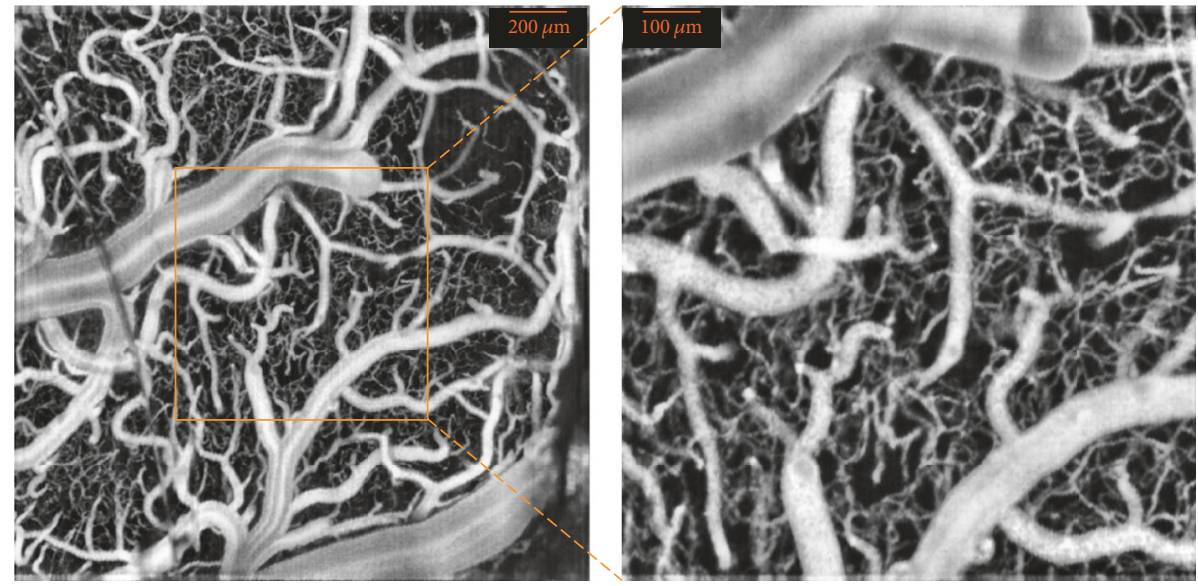

FIGURE 10: OCT angiography of capillaries in a rat brain. The imaging resolution system allows for imaging vasculature and small capillaries in the cortex of a rat. The image is the maximum intensity projection of a thickness of $\sim 220 \mu \mathrm{m}$ after averaging 5 angiography recordings [46].

declines noticeably. A combination of beam truncation and different optical aberrations can result in such an intensity drop in the imaging system.

We have successfully used this setup for in vivo brain imaging in adult rodents. The system was able to image cerebral arteries, veins, and capillaries. Figure 10 demonstrates the vasculature morphology that was captured by the device using OCT angiography $[13,14,46]$. This image shows the maximum intensity projection (MIP) obtained from a $3 \mathrm{D}$ OCT angiography that was captured from a rat. The depth that was used for MIP was from $60 \mu \mathrm{m}$ to $280 \mu \mathrm{m}$ below the brain surface. In this recording, the focal plane of the OCT scanner was positioned $\sim 100 \mu \mathrm{m}$ below the surface.

By adjusting the focal plane of the OCT, it is possible to record high-resolution OCT images at deeper regions in the brain. An example of vascular network which was captured at three different depths is demonstrated in Figure 11. In this figure, some penetrating arterioles are marked with red arrows. The circle on the image marks an arteriole that penetrates up to $500 \mu \mathrm{m}$ without branching.
Typical imaging depth of a SD-OCT system in the brain is limited to less than $1 \mathrm{~mm}$. When the camera exposure time was set to $\sim 25 \mu \mathrm{s}$, the proposed system was able to image vessels at a depth of up to $900 \mu \mathrm{m}$ (Figure 12). To the best of our knowledge, this is the deepest reported SD-OCT vascular imaging at a central wavelength of $1300 \mathrm{~nm}$.

\section{Conclusion}

This paper is aimed at assisting researchers in optimizing the optical design of spectral-domain OCT systems according to their specific needs and research goals. In this paper, a brief overview of the fundamental theory of image formation in SD-OCT scanners was presented. Then, a broad category of theoretical and practical considerations for the selection and design of optical and optomechanical components were discussed. The paper provides an analysis on each of the critical components and explains how different parameters of each piece can affect the ultimate performance of a SDOCT scanner. We also provided some details to design 

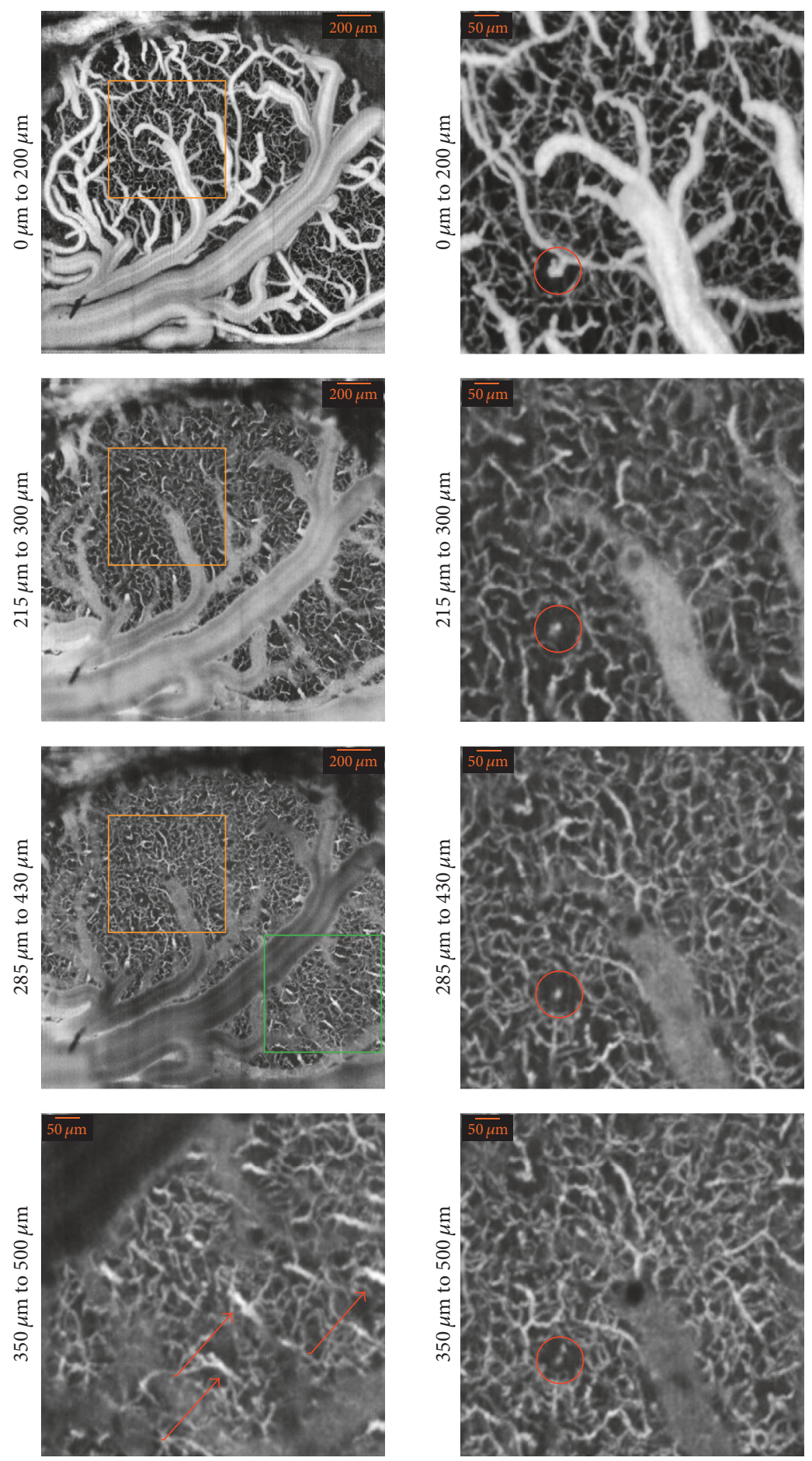

(a)

(b)

FIGURE 11: Imaging at various depths. By adjusting the position of the focal plane inside the brain, it is possible to analyze the topology and distribution of vascular networks at different depths in the brain. The red arrows mark some penetrating arterioles. The red circle on (b) depicts an arteriole that penetrates up to $500 \mu \mathrm{m}$ deep in the brain before branching.

a mechanism to combine the OCT with other imaging modalities such as fluorescence imaging or optogenetic neurostimulation technology.

The components and optical design of a custom-made $\mathrm{SD}-\mathrm{OCT}$ system that we used in our research for our in vivo brain imaging applications were discussed in further detail in this paper. This device is a relatively low cost imaging system $(\sim 34 \mathrm{~K})$ which was constructed mostly using offthe-shelf components. Making the scanner head and a few adapters requires some level of machining; however, the 

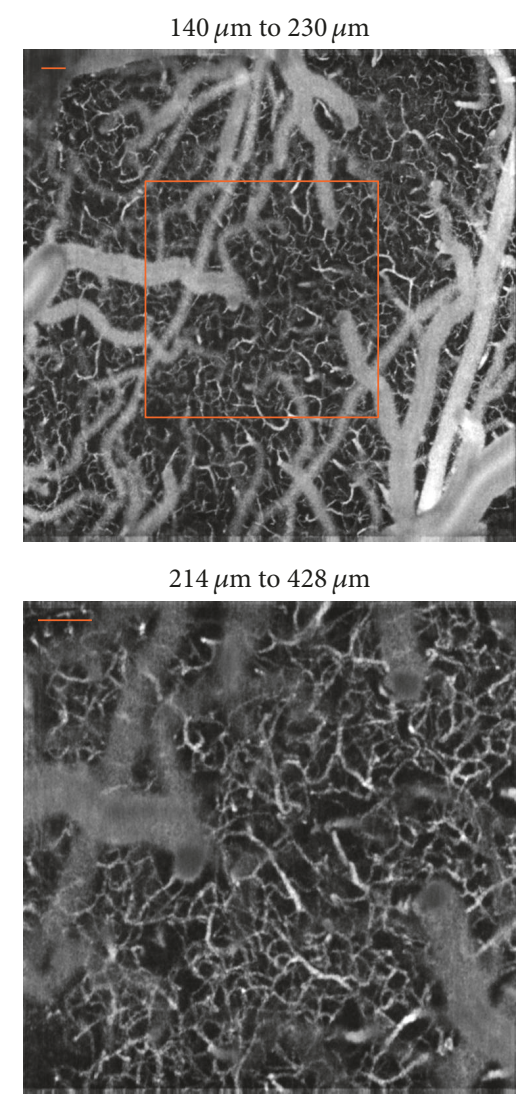

$428 \mu \mathrm{m}$ to $570 \mu \mathrm{m}$

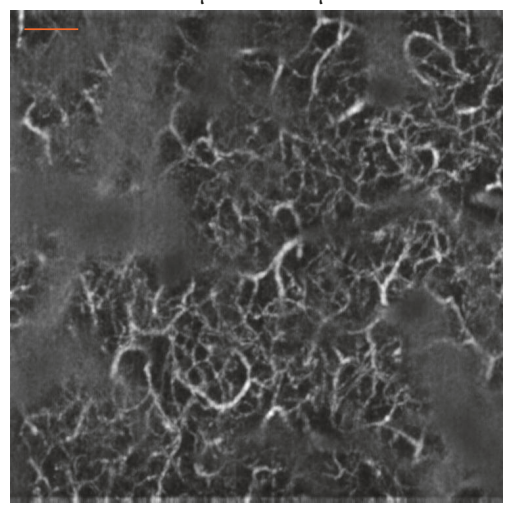

(a)

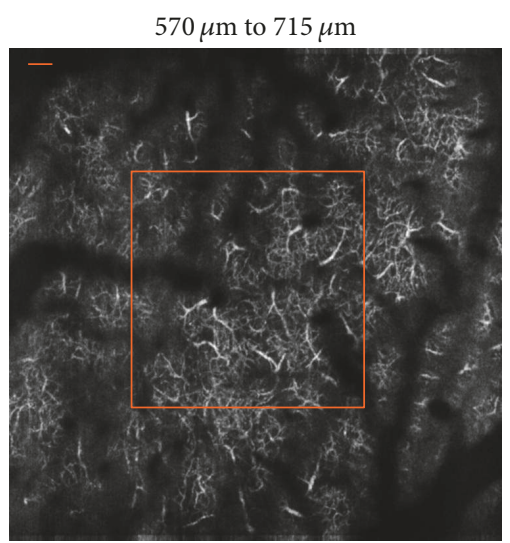

$570 \mu \mathrm{m}$ to $715 \mu \mathrm{m}$

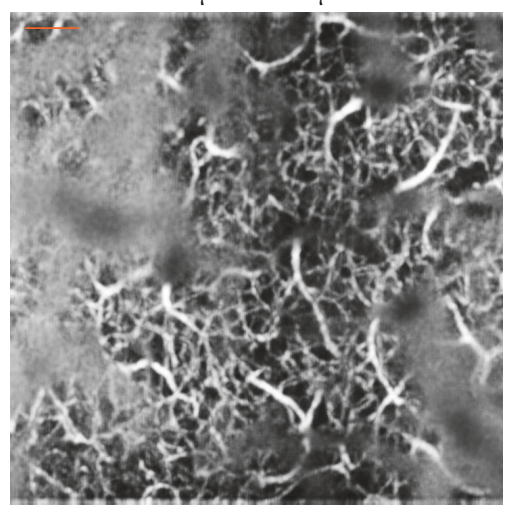

$780 \mu \mathrm{m}$ to $926 \mu \mathrm{m}$

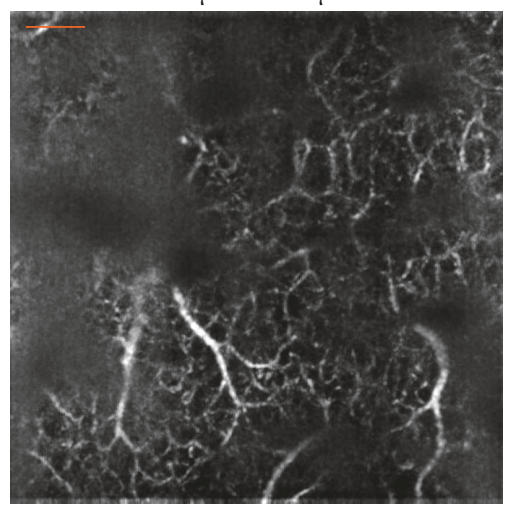

(b)

FIGURE 12: Deep brain imaging. The system is able to image vasculature up to $900 \mu \mathrm{m}$ deep in the brain of a rat. When imaging deep regions of a brain, the large pial vessels on the surface block the OCT beam and create dark and noisy areas in the OCT angiography images. (a) and (b) are obtained from the same region but at different depths. The shadow of the superficial vessels on (a) prevents imaging the capillaries at deeper regions that are shown on (b). The scale bars are $200 \mu \mathrm{m}$.

designs are fairly simple and easy to implement. Adaptation of the minimum number of custom-made parts reduced the time and cost of our system development.

Our design included the fabrication of a scan and tube lens system to incorporate a dichroic beam splitter in the path of the OCT beam and addition of a second imaging modality through the same objective lens. A simple design of a fluorescence imaging path is discussed in Figure 4.

The designed SD-OCT imaging system possesses a closeto-diffraction-limit lateral resolution and its axial resolution is comparable to the theoretical value. The system provides the diffraction limited resolution across a large FOV $(4 \mathrm{~mm}$ in diameter) without major intensity drop across this area. In the design, we considered the possibility of two different optical paths in the reference arm. Therefore, it is possible to change the objective lens during the experiment. Alternating between two objectives may require some adjustments in the objective collar.

Although the presented design and discussions facilitate the process of building new custom-made SD-OCT scanners, 
the authors would like to emphasize that such system development projects are delicate time-consuming efforts. Proper alignment of the components is crucial to achieve the diffraction limited performance to obtain theoretical specifications. While for an experienced person the process can take a few days, for a nonexpert designer with no or minimum background in optical design, the process can be tedious and much more time-consuming to assemble and align the same platform. The Supplementary Materials summarize some steps to help nonexpert users achieve proper optical alignment.

Obviously, such an imaging system requires a proper data acquisition system and analysis software to become a fully functional setup. While for two-photon microscopy there are already open-source software packages to operate a newly developed system, similar software packages are not available for custom-made OCT scanners. Development of publicly available software packages that are modular and adjustable to general needs in the field of OCT imaging seems necessary.

\section{Conflicts of Interest}

The authors declare that there are no conflicts of interest regarding the publication of this paper.

\section{Acknowledgments}

This research was supported by the Army Research Office (ARO) Grant no. 68750-LS-II, the National Science Foundation (NSF) Career Award Grant no. 1454300, the Brain and Behavior Research Foundation (NARSAD) Young Investigator Award Grants nos. 20610 and 23620, and the University of Wisconsin Research Growth Initiative (RGI) Grants nos. $101 \mathrm{X} 213$ and 101X254.

\section{Supplementary Materials}

In the Supplementary Materials of this paper, we discuss some practical consideration for the alignment of optical components to help unexperienced users achieve a highperformance optical setup. (Supplementary Materials)

\section{References}

[1] A. F. Fercher, C. K. Hitzenberger, G. Kamp, and S. Y. ElZaiat, "Measurement of intraocular distances by backscattering spectral interferometry," Optics Communications, vol. 117, no. 12, pp. 43-48, 1995.

[2] J. Men, Y. Huang, J. Solanki et al., "Optical Coherence Tomography for Brain Imaging and Developmental Biology," IEEE Journal of Selected Topics in Quantum Electronics, vol. 22, no. 4, 2016.

[3] C. Magnain, J. C. Augustinack, M. Reuter et al., "Blockface histology with optical coherence tomography: a comparison with Nissl staining," NeuroImage, vol. 84, pp. 524-533, 2014.

[4] V. J. Srinivasan, H. Radhakrishnan, J. Y. Jiang, S. Barry, and A. E. Cable, "Optical Coherence Microscopy for deep tissue imaging of the cerebral cortex with intrinsic contrast," Optics Express, vol. 20, no. 3, pp. 2220-2239, 2012.
[5] C. Magnain, J. C. Augustinack, E. Konukoglu et al., "Optical coherence tomography visualizes neurons in human entorhinal cortex," Neurophotonics, vol. 2, no. 1, Article ID 14063RR, 2015.

[6] N. A. Nassif, B. Cense, B. H. Park et al., "In vivo high-resolution video-rate spectral-domain optical coherence tomography of the human retina and optic nerve," Optics Express, vol. 12, no. 3, pp. 367-376, 2004.

[7] M. Wojtkowski, V. Srinivasan, J. G. Fujimoto et al., “Threedimensional retinal imaging with high-speed ultrahighresolution optical coherence tomography," Ophthalmology, vol. 112, no. 10, pp. 1734-1746, 2005.

[8] Y. Ikuno and Y. Tano, "Retinal and choroidal biometry in highly myopic eyes with spectral-domain optical coherence tomography," Investigative Ophthalmology \& Visual Science, vol. 50, no. 8, pp. 3876-3880, 2009.

[9] V. J. Srinivasan, Y. Chen, J. S. Duker, and J. G. Fujimoto, "In vivo functional imaging of intrinsic scattering changes in the human retina with high-speed ultrahigh resolution OCT," Optics Express, vol. 17, no. 5, pp. 3861-3877, 2009.

[10] Q. Zhang, R. Lu, B. Wang, J. D. Messinger, C. A. Curcio, and X. Yao, "Functional optical coherence tomography enables in vivo physiological assessment of retinal rod and cone photoreceptors," Scientific Reports, vol. 5, article no. 9595, 2015.

[11] S. Makita, Y. Hong, M. Yamanari, T. Yatagai, and Y. Yasuno, "Optical coherence angiography," Optics Express, vol. 14, no. 17, pp. 7821-7840, 2006.

[12] R. K. Wang, S. L. Jacques, Z. Ma, S. Hurst, S. R. Hanson, and A. Gruber, "Three dimensional optical angiography," Optics Express, vol. 15, no. 7, pp. 4083-4097, 2007.

[13] B. J. Vakoc, R. M. Lanning, J. A. Tyrrell et al., "Threedimensional microscopy of the tumor microenvironment in vivo using optical frequency domain imaging," Nature Medicine, vol. 15, no. 10, pp. 1219-1223, 2009.

[14] V. J. Srinivasan, J. Y. Jiang, M. A. Yaseen et al., "Rapid volumetric angiography of cortical microvasculature with optical coherence tomography," Optics Expresss, vol. 35, no. 1, pp. 43-45, 2010.

[15] Y. Zhao, Z. Chen, C. Saxer, S. Xiang, J. F. De Boer, and J. S. Nelson, "Phase-resolved optical coherence tomography and optical Doppler tomography for imaging blood flow in human skin with fast scanning speed and high velocity sensitivity," Optics Expresss, vol. 25, no. 2, pp. 114-116, 2000.

[16] R. Leitgeb, L. Schmetterer, M. Wojtkowski, C. K. Hitzenberger, M. Sticker, and A. F. Fercher, "Flow velocity measurements by frequency domain short coherence interferometry," in Proceedings of the Coherence Domain Optical Methods in Biomedical Science and Clinical Applications VI, pp. 16-21, USA, January 2002.

[17] B. R. White, M. C. Pierce, N. Nassif et al., "In vivo dynamic human retinal blood flow imaging using ultra-high-speed spectral domain optical doppler tomography," Optics Express, vol. 11, no. 25, pp. 3490-3497, 2003.

[18] V. X. D. Yang, M. L. Gordon, B. Qi et al., "High speed, wide velocity dynamic range Doppler optical coherence tomography (Part I): System design, signal processing, and performance," Optics Express, vol. 11, no. 7, pp. 794-809, 2003.

[19] U. Morgner, W. Drexler, F. X. Kärtner et al., "Spectroscopic optical coherence tomography," Optics Expresss, vol. 25, no. 2, pp. 111-113, 2000.

[20] D. J. Faber, E. G. Mik, M. C. G. Aalders, and T. G. Van Leeuwen, "Toward assessment of blood oxygen saturation by spectroscopic optical coherence tomography," Optics Expresss, vol. 30, no. 9, pp. 1015-1017, 2005. 
[21] F. E. Robles, C. Wilson, G. Grant, and A. Wax, "Molecular imaging true-colour spectroscopic optical coherence tomography," Nature Photonics, vol. 5, no. 12, pp. 744-747, 2011.

[22] M. R. Hee, D. Huang, E. A. Swanson, and J. G. Fujimoto, "Polarization-sensitive low-coherence reflectometer for birefringence characterization and ranging," Journal of the Optical Society of America B: Optical Physics, vol. 9, no. 6, pp. 903-908, 1992.

[23] J. F. De Boer, T. E. Milner, M. J. C. Van Gemert, and J. S. Nelson, "Two-dimensional birefringence imaging in biological tissue by polarization-sensitive optical coherence tomography," Optics Expresss, vol. 22, no. 12, pp. 934-936, 1997.

[24] J. F. De Boer, T. E. Milner, and J. Stuart Nelson, "Determination of the depth-resolved Stokes parameters of light backscattered from turbid media by use of polarization-sensitive optical coherence tomography," Optics Expresss, vol. 24, no. 5, pp. 300302, 1999.

[25] E. Götzinger, M. Pircher, and C. K. Hitzenberger, "High speed spectral domain polarization sensitive optical coherence tomography of the human retina," Optics Express, vol. 13, no. 25, pp. 10217-10229, 2005.

[26] B. H. Park, M. C. Pierce, B. Cense et al., "Real-time fiber-based multi-functional spectral-domain optical coherence tomography at 1.3 $\mu \mathrm{m}$," Optics Express, vol. 13, no. 11, pp. 3931-3944, 2005.

[27] M. Yamanari, S. Makita, V. D. Madjarova, T. Yatagai, and Y. Yasuno, "Fiber-based polarization-sensitive Fourier domain optical coherence tomography using B-scan-oriented polarization modulation method," Optics Express, vol. 14, no. 14, pp. 6502-6515, 2006.

[28] B. Baumann, E. Götzinger, M. Pircher et al., "Segmentation and quantification of retinal lesions in age-related macular degeneration using polarization-sensitive optical coherence tomography," Journal of Biomedical Optics, vol. 15, no. 6, Article ID 061704, 2010.

[29] J. M. Schmitt, "OCT elastography: Imaging microscopic deformation and strain of tissue," Optics Express, vol. 3, no. 6, pp. 199211, 1998.

[30] J. Rogowska, N. A. Patel, J. G. Fujimoto, and M. E. Brezinski, "Optical coherence tomographic elastography technique for measuring deformation and strain of atherosclerotic tissues," Heart, vol. 90, no. 5, pp. 556-562, 2004.

[31] S. G. Adie, X. Liang, B. F. Kennedy, R. John, D. D. Sampson, and S. A. Boppart, "Spectroscopic optical coherence elastography," Optics Express, vol. 18, no. 25, pp. 25519-25534, 2010.

[32] B. F. Kennedy, X. Liang, S. G. Adie et al., "In vivo threedimensional optical coherence elastography," Optics Express, vol. 19, no. 7, pp. 6623-6634, 2011.

[33] W. Drexler and J. G. Fujimoto, Optical Coherence Tomography, Springer International Publishing, Cham, 1st edition, 2008.

[34] J. Kim, W. Brown, J. R. Maher, H. Levinson, and A. Wax, "Functional optical coherence tomography: Principles and progress," Physics in Medicine and Biology, vol. 60, no. 10, pp. R211-R237, 2015.

[35] C. Dorrer, N. Belabas, J.-P. Likforman, and M. Joffre, "Spectral resolution and sampling issues in fourier-transform spectral interferometry," Journal of the Optical Society of America B: Optical Physics, vol. 17, no. 10, pp. 1795-1802, 2000.

[36] M. Wojtkowski, R. Leitgeb, A. Kowalczyk, T. Bajraszewski, and A. F. Fercher, "In vivo human retinal imaging by Fourier domain optical coherence tomography," Journal of Biomedical Optics, vol. 7, no. 3, pp. 457-463, 2002.
[37] M. A. Choma, M. V. Sarunic, C. Yang, and J. A. Izatt, "Sensitivity advantage of swept source and Fourier domain optical coherence tomography," Optics Express, vol. 11, no. 18, pp. 2183-2189, 2003.

[38] S. H. Yun, G. J. Tearney, J. F. De Boer, N. Iftimia, and B. E. Bouma, "High-speed optical frequency-domain imaging," Optics Express, vol. 11, no. 22, pp. 2953-2963, 2003.

[39] A. F. Fercher, W. Drexler, C. K. Hitzenberger, and T. Lasser, "Optical coherence tomography-principles and applications," Reports on Progress in Physics, vol. 66, no. 2, pp. 239-303, 2003.

[40] R. Leitgeb, C. K. Hitzenberger, and A. F. Fercher, "Performance of fourier domain vs. Time domain optical coherence tomography," Optics Express, vol. 11, no. 8, pp. 889-894, 2003.

[41] J. F. de Boer, B. Cense, B. H. Park, M. C. Pierce, G. J. Tearney, and B. E. Bouma, "Improved signal-to-noise ratio in spectraldomain compared with time-domain optical coherence tomography," Optics Expresss, vol. 28, no. 21, pp. 2067-2069, 2003.

[42] B. Cense, N. A. Nassif, T. C. Chen et al., "Ultrahigh-resolution high-speed retinal imaging using spectral-domain optical coherence tomography," Optics Express, vol. 12, no. 11, pp. 24352447, 2004.

[43] M. Wojtkowski, V. J. Srinivasan, T. H. Ko, J. G. Fujimoto, A. Kowalczyk, and J. S. Duker, "Ultrahigh-resolution, high-speed, Fourier domain optical coherence tomography and methods for dispersion compensation," Optics Express, vol. 12, no. 11, pp. 2404-2422, 2004.

[44] B. F. Kennedy, K. M. Kennedy, and D. D. Sampson, "A review of optical coherence elastography: Fundamentals, techniques and prospects," IEEE Journal of Selected Topics in Quantum Electronics, vol. 20, no. 2, 2014.

[45] "A quickly made model of a microscope stand," https://grabcad .com/library/microscope-stand-1.

[46] F. Atry, S. Frye, T. J. Richner et al., "Monitoring cerebral hemodynamics following optogenetic stimulation via optical coherence tomography," IEEE Transactions on Biomedical Engineering, vol. 62, no. 2, pp. 766-773, 2015.

[47] D. Huang, E. A. Swanson, C. P. Lin et al., "Optical coherence tomography," Science, vol. 254, no. 5035, pp. 1178-1181, 1991.

[48] J. A. Izatt, M. D. Kulkarni, H.-W. Wang, K. Kobayashi, and M. V. Sivak Jr., "Optical coherence tomography and microscopy in gastrointestinal tissues," IEEE Journal of Selected Topics in Quantum Electronics, vol. 2, no. 4, pp. 1017-1028, 1996.

[49] Y.-K. Kim and Y. P. Kim, "High-speed time-domain optical coherence tomography with an imaging speed of ten frames per second with 2000 A-scan," Optical Engineering, vol. 49, no. 5, Article ID 055601, 2010.

[50] G. Häusler and M. W. Lindner, "Coherence radar' and 'spectral radar'-new tools for dermatological diagnosis," Journal of Biomedical Optics, vol. 3, no. 1, pp. 21-31, 1998.

[51] J. M. Schmitt, "Optical Coherence Tomography (OCT): a review," IEEE Journal of Selected Topics in Quantum Electronics, vol. 5, no. 4, pp. 1205-1215, 1999.

[52] P. H. Tomlins and R. K. Wang, "Theory, developments and applications of optical coherence tomography," Journal of Physics D: Applied Physics, vol. 38, no. 15, pp. 2519-2535, 2005.

[53] J. Kalkman, "Fourier-Domain Optical Coherence Tomography Signal Analysis and Numerical Modeling," International Journal of Optics, vol. 2017, Article ID 9586067, 2017.

[54] F. E. Robles, S. Chowdhury, and A. Wax, "Assessing hemoglobin concentration using spectroscopic optical coherence tomography for feasibility of tissue diagnosticcs," Biomedical Optics Express, vol. 1, no. 1, pp. 310-317, 2010. 
[55] J. Yi and X. Li, "Estimation of oxygen saturation from erythrocytes by high-resolution spectroscopic optical coherence tomography," Optics Expresss, vol. 35, no. 12, pp. 2094-2096, 2010.

[56] J. Yi, Q. Wei, W. Liu, V. Backman, and H. F. Zhang, "Visiblelight optical coherence tomography for retinal oximetry," Optics Expresss, vol. 38, no. 11, pp. 1796-1798, 2013.

[57] S. P. Chong, C. W. Merkle, D. F. Cooke et al., "Noninvasive, in vivo imaging of subcortical mouse brain regions with $1.7 \mu \mathrm{m}$ optical coherence tomography," Optics Express, vol. 40, no. 21, pp. 4911-4914, 2015.

[58] S. Ishida, N. Nishizawa, T. Ohta, and K. Itoh, "Ultrahighresolution optical coherence tomography in $1.7 \mu \mathrm{m}$ region with fiber laser supercontinuum in low-water-absorption samples," Applied Physics Express, vol. 4, no. 5, 2011.

[59] S. Ishida and N. Nishizawa, "Quantitative comparison of contrast and imaging depth of ultrahigh-resolution optical coherence tomography images in 800-1700 nm wavelength region," Biomedical Optics Express, vol. 3, no. 2, pp. 282-294, 2012.

[60] Y. Hattori, H. Kawagoe, Y. Ando, M. Yamanaka, and N. Nishizawa, "High-speed ultrahigh-resolution spectral domain optical coherence tomography using high-power supercontinuum at $0.8 \mu \mathrm{m}$ wavelength," Applied Physics Express, vol. 8, no. 8, Article ID 082501, 2015.

[61] M. Szkulmowski, M. Wojtkowski, T. Bajraszewski et al., "Quality improvement for high resolution in vivo images by spectral domain optical coherence tomography with supercontinuum source," Optics Communications, vol. 246, no. 4-6, pp. 569-578, 2005.

[62] J. Barrick, A. Doblas, M. R. Gardner, P. R. Sears, L. E. Ostrowski, and A. L. Oldenburg, "High-speed and high-sensitivity parallel spectraldomain optical coherence tomography using a supercontinuum light source," Optics Expresss, vol. 41, no. 24, pp. 5620-5623, 2016.

[63] Z. Hu and A. M. Rollins, "Theory of two beam interference with arbitrary spectra," Optics Express, vol. 14, no. 26, pp. 12751-12759, 2006.

[64] Z. Hu, Y. Pan, and A. M. Rollins, "Analytical model of spectrometer-based two-beam spectral interferometry," Applied Optics, vol. 46, no. 35, pp. 8499-8505, 2007.

[65] Z. Hu and A. M. Rolling, "Quasi-telecentric optical design of a microscope-compatible OCT scanner," Optics Express, vol. 13, no. 17, pp. 6407-6415, 2005.

[66] E. H. K. Stelzer, The Intermediate Optical System of LaserScanning Confocal Microscopes, Springer US, Boston, MA, 2006.

[67] A. Negrean and H. D. Mansvelder, "Optimal lens design and use in laser-scanning microscopy," Biomedical Optics Express, vol. 5, no. 5, pp. 1588-1609, 2014.

[68] M. Hafez, T. Sidler, and R.-P. Salathé, "Study of the beam path distortion profiles generated by a two-axis tilt single-mirror laser scanner," Optical Engineering, vol. 42, no. 4, pp. 1048-1057, 2003.

[69] J. Y. Kim, C. Lee, K. Park, G. Lim, and C. Kim, "Fast opticalresolution photoacoustic microscopy using a 2-axis waterproofing MEMS scanner," Scientific Reports, vol. 5, article no. 7932, 2015.

[70] B. E. A. Saleh and M. C. Teich, Fundamentals of Photonics, Wiley-Interscience, 1991.

[71] R. A. Leitgeb, M. Villiger, A. H. Bachmann, and L. Steinmann, "Extended focus depth for Fourier domain optical coherence microscopy," Optics Expresss, vol. 31, no. 16, pp. 2450-2452, 2006.
[72] K.-S. Lee and J. P. Rolland, "Bessel beam spectral-domain highresolution optical coherence tomography with micro-optic axicon providing extended focusing range," Optics Expresss, vol. 33, no. 15, pp. 1696-1698, 2008.

[73] S. Tamborski, H. C. Lyu, H. Dolezyczek et al., "Extended-focus optical coherence microscopy for high-resolution imaging of the murine brain," Biomedical Optics Express, vol. 7, no. 11, Article ID \#269710, pp. 4400-4414, 2016.

[74] F. Atry and R. Pashaie, "Analysis of intermediary scan-lens and tube-lens mechanisms for optical coherence tomography," Applied Optics, vol. 55, no. 4, pp. 646-653, 2016.

[75] T. Bajraszewski, M. Wojtkowski, M. Szkulmowski, A. Szkulmowska, R. Huber, and A. Kowalczyk, "Improved spectral optical coherence tomography using optical frequency comb," Optics Express, vol. 16, no. 6, pp. 4163-4176, 2008.

[76] V. Fathipour, A. Bonakdar, and H. Mohseni, "Advances on Sensitive Electron-Injection Based Cameras for Low-Flux, Short-Wave Infrared Applications," Frontiers in Materials, vol. 3, no. 33, 2016.

[77] V. M. Gelikonov, G. V. Gelikonov, and P. A. Shilyagin, "Linearwavenumber spectrometer for high-speed spectral-domain optical coherence tomography," Optics and Spectroscopy (English translation of Optika i Spektroskopiya), vol. 106, no. 3, pp. 459-465, 2009.

[78] J. Kim, D. T. Miller, E. Kim, S. Oh, J. Oh, and T. E. Milner, "Optical coherence tomography speckle reduction by a partially spatially coherent source," Journal of Biomedical Optics, vol. 10, no. 6, Article ID 064034, 2005.

[79] A.-H. Dhalla, J. V. Migacz, and J. A. Izatt, "Crosstalk rejection in parallel optical coherence tomography using spatially incoherent illumination with partially coherent sources," Optics Expresss, vol. 35, no. 13, pp. 2305-2307, 2010.

[80] M. Sato, D. Saito, K. Shouji, R. Kurotani, H. Abe, and I. Nishidate, "Ultrathin forward-imaging short multimode fiber probe for full-field optical coherence microscopy," Optics Communications, vol. 381, pp. 296-308, 2016.

[81] P. Eugui, D. J. Harper, A. Lichtenegger et al., "Multimode fiber for angle-resolved optical coherence tomography," in Proceedings of the Bio-Optics: Design and Application, p. BoM4A.3, San Diego, California, USA, 2017. 

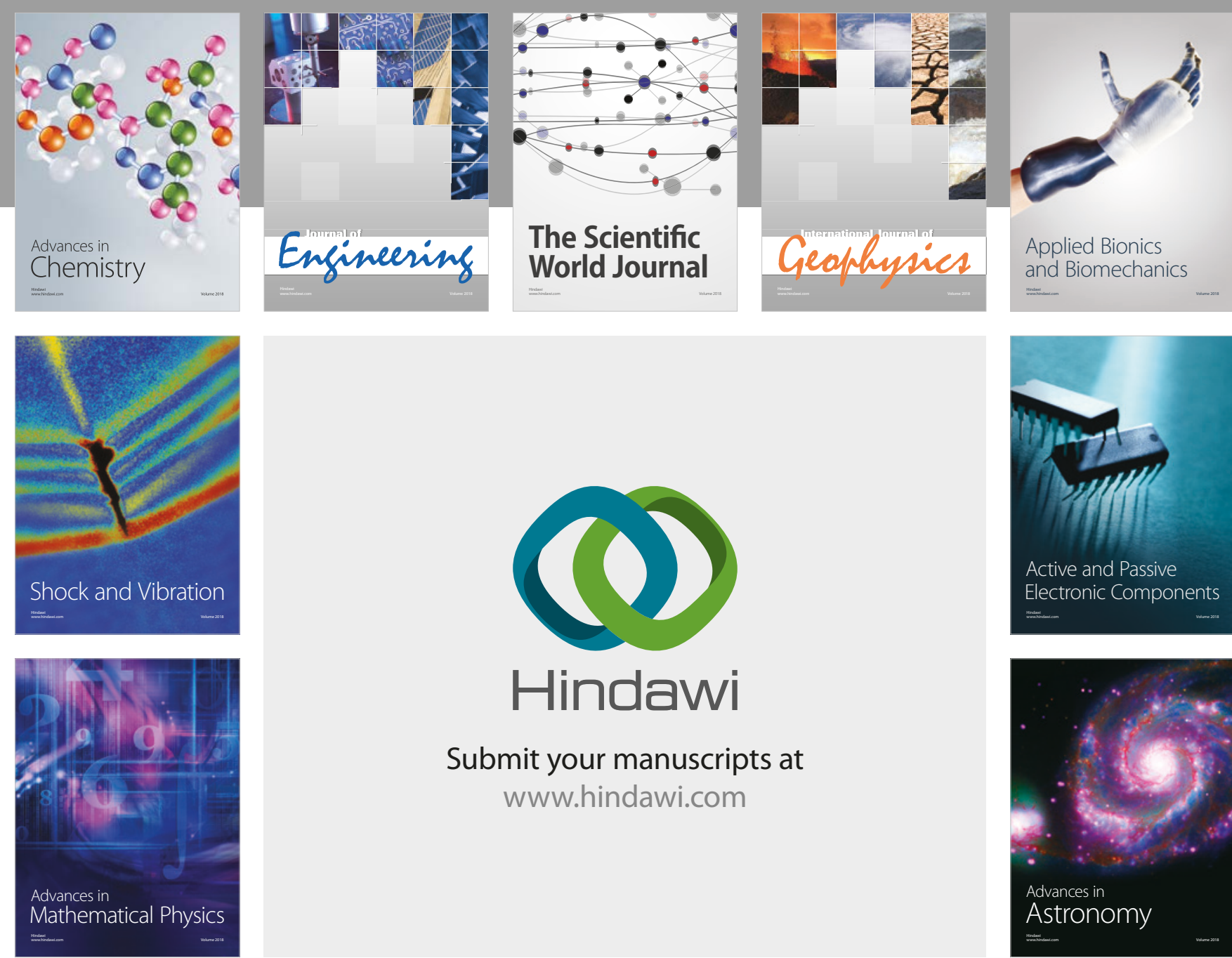

Submit your manuscripts at

www.hindawi.com

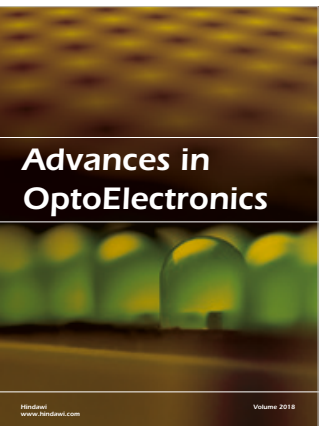

\section{Rotcting Machinery}
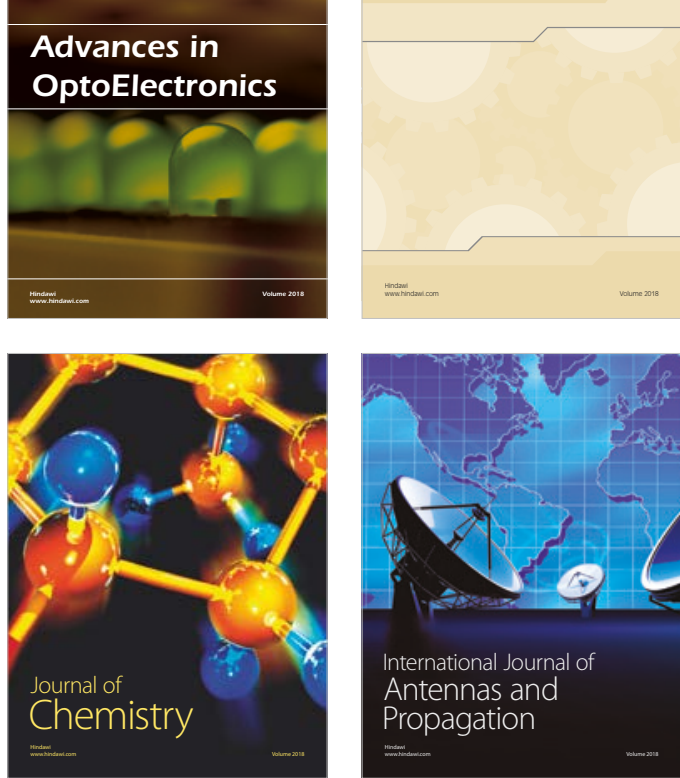

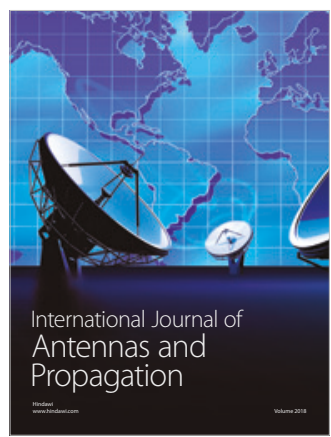

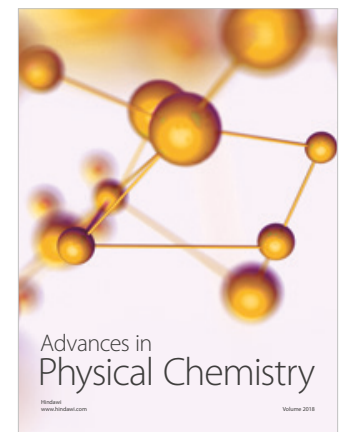

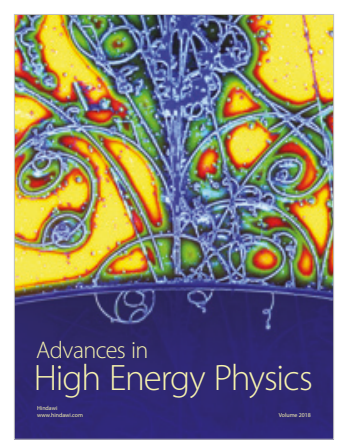

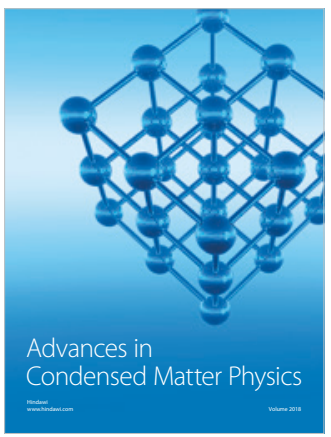

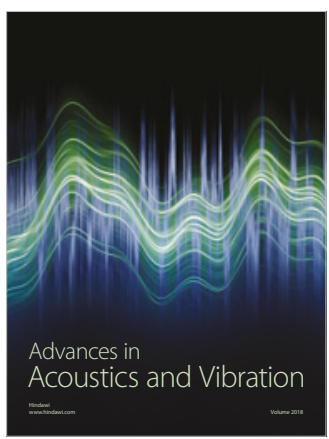

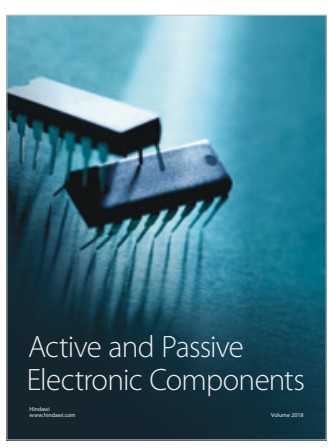
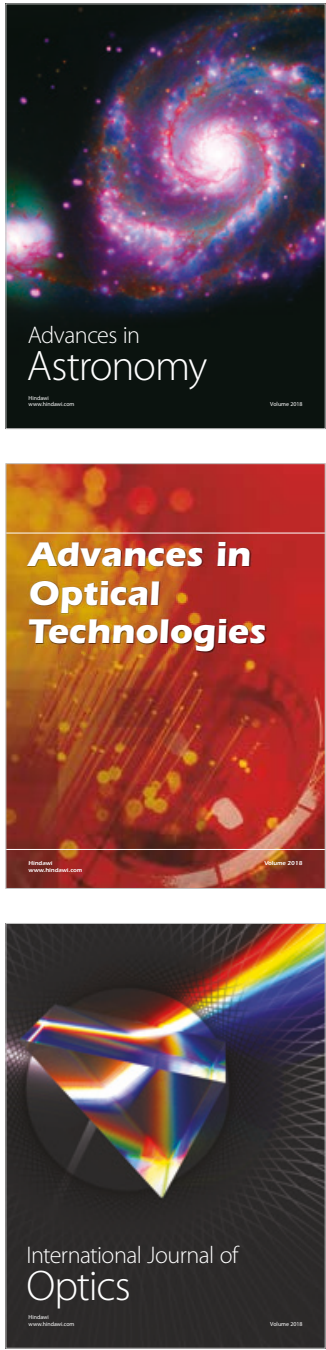